\title{
Mindestlohnregelungen im Maler- und Lackiererhandwerk: Eine Wirkungsanalyse
}

\author{
Bernhard Boockmann • Michael Neumann • \\ Pia Rattenhuber
}

Angenommen: 2. Oktober 2012 / Online publiziert: 14. November 2012

(C) Institut für Arbeitsmarkt- und Berufsforschung 2012

Zusammenfassung Die Einführung des Mindestlohns im deutschen Maler- und Lackiererhandwerk im Jahr 2003 wird als natürliches Experiment genutzt, um die Effekte von Mindestlöhnen auf Löhne und Beschäftigung $\mathrm{zu}$ untersuchen. Wir finden einen signifikant positiven Effekt auf den durchschnittlichen Stundenlohn der gelernten Beschäftigten in Ostdeutschland, nicht aber in Westdeutschland und nicht für ungelernte Arbeitskräfte. Dieser Befund entspricht der unterschiedlichen Eingriffsintensität der Mindestlöhne. Signifikant positive oder negative Beschäftigungseffekte werden nicht gefunden. Neuere Befragungsergebnisse deuten auf die fortbestehende Relevanz des Mindestlohns in Ostdeutschland hin.

Schlüsselwörter Mindestlohn · Beschäftigung · Löhne · Arbeitsmarktregulierung · Maler- und Lackiererhandwerk

\section{JEL Klassifikationen J38 · J31}

Für die in diesem Beitrag publizierten Ergebnisse und Schlussfolgerungen zeichnen ausschließlich die Autorinnen und Autoren verantwortlich. Sie geben nicht notwendigerweise die Position des Bundesministerium für Arbeit und Soziales wieder.

B. Boockmann $(\bowtie)$

Institut für Angewandte Wirtschaftsforschung, IAW, Tübingen, Deutschland

e-mail: bernhard.boockmann@iaw.edu

B. Boockmann

Universität Tübingen, Tübingen, Deutschland

B. Boockmann

IZA, Bonn, Deutschland

M. Neumann · P. Rattenhuber

Deutsches Institut für Wirtschaftsforschung, DIW, Berlin,

Deutschland

\section{An impact analysis of the minimum wage in the German painter and varnisher trades}

\begin{abstract}
The introduction of the minimum wage in the German painter and varnisher trade in 2003 is used as a natural experiment to study the effects of minimum wages on wages and employment. We find a significant positive effect on the average hourly wage of skilled workers in East Germany, but not in West Germany and not for unskilled workers. This finding corresponds to the differences in the strength of the minimum wage across these groups. No significant positive or negative employment effects are found. Recent survey results indicate the continuing relevance of the minimum wage in East Germany.
\end{abstract}

\section{Einleitung}

Im deutschen Maler- und Lackiererhandwerk wurden erstmals zum 1. Dezember 2003 Mindestlöhne nach dem Arbeitnehmer-Entsendegesetz eingeführt. Damit folgte diese Handwerksbranche dem Beispiel anderer Branchen des Bausektors wie dem Bauhauptgewerbe, dem Elektrohandwerk und dem Dachdeckerhandwerk, in denen Mindestlöhne schon seit dem Jahr 1997 bestanden. Die Mindestlöhne im Maler- und Lackiererhandwerk sind nach Ost- und Westdeutschland differenziert und haben derzeit in Westdeutschland zwei Stufen für gelernte und ungelernte Arbeitskräfte, während diese Unterscheidung seit 2009 in Ostdeutschland entfallen ist.

Im Folgenden werden wichtige Ergebnisse aus dem Abschlussbericht des Instituts für Angewandte Wirtschaftsforschung (IAW) zur Evaluation der Mindestlöhne im Malerund Lackiererhandwerk wiedergegeben, die im Auftrag der 
Abb. 1 Entwicklung der Beschäftigtenzahlen in der Berufsgruppe Maler und Lackierer. Quelle: IAB, Berufe im Spiegel der Statistik

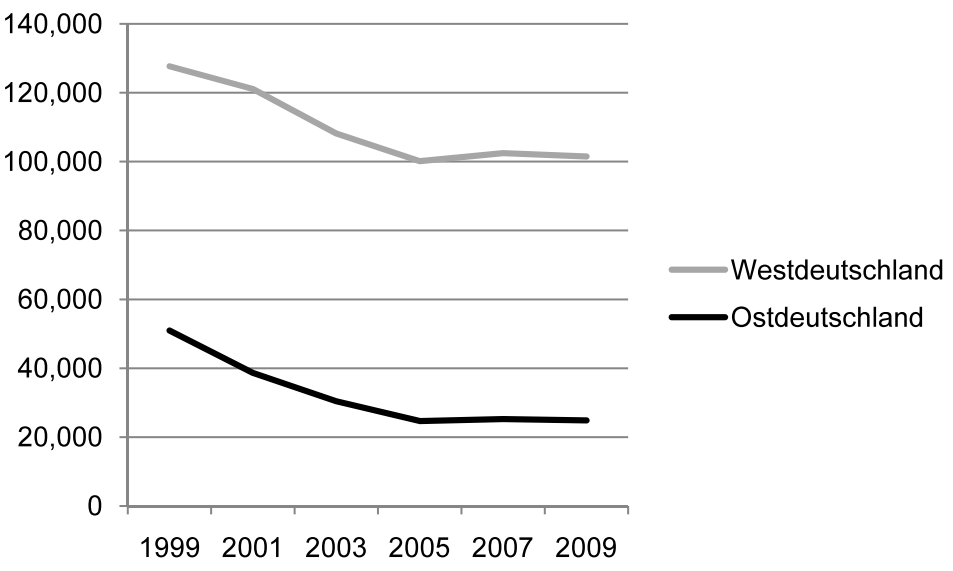

Bundesregierung durchgeführt wurde. ${ }^{1}$ Der Abschlussbericht (IAW 2011a) enthält weitere Ergebnisse, die hier nicht im Einzelnen aufgeführt werden können. Gegenüber dem Bericht wurden für dieses Papier keine neuen Schätzungen zu den Wirkungen der Mindestlöhne durchgeführt.

Die Einführung des Mindestlohns im Jahr 2003 traf die Branche in einer Situation einer seit Mitte der 1990er Jahr rückläufigen Nachfrage, die sie mit allen Branchen des Bausektors teilte. Obwohl der Rückgang des Neubauvolumens teilweise durch eine Zunahme der Aufträge im Gebäudebestand kompensiert werden konnte, ging die Zahl der Beschäftigten in den Maler- und Lackiererberufen bis nach 2003 deutlich zurück (siehe Abb. 1). Dies betraf vor allem Ostdeutschland. In den Folgejahren verbesserte sich die Situation auf dem Absatzmarkt, ohne dass sich dies in einem merklichen Zuwachs in der Anzahl der Beschäftigten niederschlug. Auch die Wirtschaftskrise 2008-2009 hatte keine gravierenden negativen Auswirkungen auf das Geschäftsvolumen, wozu die Maßnahmen des Konjunkturpakets II der Bundesregierung erheblich beitrugen.

Evaluationsergebnisse für die Mindestlöhne im Malerund Lackiererhandwerk lagen vor der durch die Bundesregierung beauftragten Evaluation nicht vor. Lediglich für das Bauhauptgewerbe waren Evaluationsergebnisse verfügbar. Diese folgten dem Ansatz, auf der Basis mikroökonomischer Daten für einzelne Betriebe und Beschäftigte die Treatment-Effekte der Mindestlöhne zu schätzen. Die Analysen von König und Möller (2008) ergeben, dass die Mindestlöhne in Ostdeutschland zu einer stärkeren Anhebung der Löhne im unteren Bereich der Lohnverteilung geführt haben als in Westdeutschland. Während für Westdeutschland insignifikante oder schwach signifikante positive Auswirkungen auf die Beschäftigung geschätzt werden, sind

\footnotetext{
${ }^{1}$ Durch das IAW wurde die Wirkung von Mindestlöhnen außer in den Branchen Maler- und Lackiererhandwerk und Pflege (siehe den Beitrag von Verbeek und Harsch in dieser Ausgabe) auch im Elektrohandwerk analysiert. Die Ergebnisse hierzu sind in IAW (2011b) sowie in Boockmann et al. (2012) enthalten.
}

die geschätzten Beschäftigungswirkungen für Ostdeutschland signifikant negativ. Dieser negative Effekt auf die Beschäftigung im ostdeutschen Bauhauptgewerbe wurde in der durch die Bundesregierung beauftragten Evaluation allerdings nicht bestätigt (IAB et al. 2011). Weitere Ergebnisse von Rattenhuber (2011) zeigen, dass die Mindestlöhne eine Wirkung nicht nur auf die Löhne der unmittelbar Betroffenen, sondern auch auf Beschäftigte in höheren Quantilen der Lohnverteilung hatten. Die Ergebnisse von Müller (2010) implizieren einen Beschäftigungsverlust infolge der Mindestlöhne von ungefähr $4 \%$ bis $5 \%$ in Ostdeutschland, der zwischen unterschiedlichen Gruppen von Beschäftigten (nach Alter, Qualifikationsgruppen, Betriebsgrößenklassen usw.) recht unterschiedlich ausfällt. Für Westdeutschland werden nur geringe Beschäftigungsverluste ermittelt. Insgesamt ist die Entwicklung des Maler- und Lackiererhandwerks so unterschiedlich von derjenigen des Bauhauptgewerbes, dass diese Ergebnisse nicht einfach übertragen werden können.

Im Folgenden stellen wir die Ergebnisse der quantitativen Untersuchungsschritte der Evaluation vor, während für die umfangreiche qualitative Evidenz auf den Bericht (IAW 2011a) verwiesen wird. Eine Besonderheit des Mindestlohns im Maler- und Lackiererhandwerk ist die Differenzierung zwischen ,ungelernten“ und ,gelernten“ Beschäftigten. Dies hat für die Evaluation den Vorteil, dass in Kombination mit der Differenzierung zwischen Ostund Westdeutschland insgesamt vier natürliche Experimente vorliegen, die sich in der betroffenen Gruppe an Beschäftigten und in der Eingriffsintensität unterscheiden.

Zunächst wird der Mindestlohn im Maler- und Lackiererhandwerk beschrieben. Abschnitt 3 stellt den Evaluationsansatz und die Datengrundlage vor. Abschnitt 4 enthält eine deskriptive Analyse der Entwicklung der Löhne sowie die geschätzten Wirkungen der Mindestlöhne auf die Durchschnittslöhne im Maler- und Lackiererhandwerk. Die Beschäftigungswirkungen sind Gegenstand von Abschn. 5. Die Ergebnisse der Wirkungsanalyse beziehen sich auf die Einführung der Mindestlöhne im Jahr 2003. Ergänzend werden 
Abb. 2 Höhe des Mindestlohns im Maler- und

Lackiererhandwerk -

Westdeutschland. Quelle:

Tarifvertrag Mindestlohn für das

Maler- und Lackiererhandwerk

in Deutschland (2003-2011)

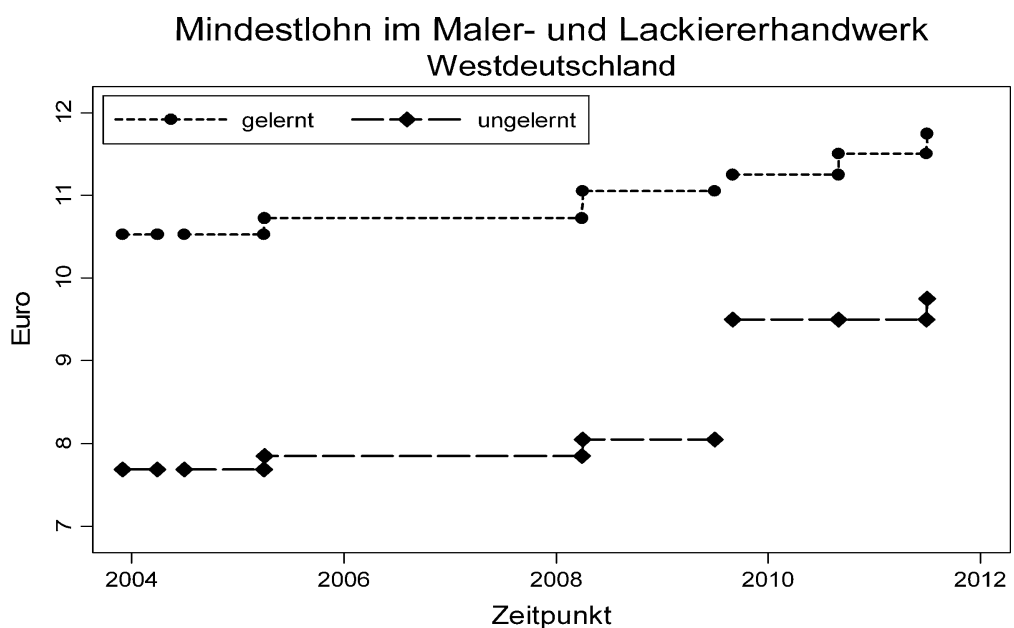

deshalb in Abschn. 6 Ergebnisse einer Befragung unter ca. 800 Betrieben des Maler- und Lackiererhandwerks aus dem Jahr 2011 vorgestellt. Sie dienen auch dazu, die Ergebnisse der quantitativen Evaluation besser zu interpretieren.

\section{Der Mindestlohn im Maler- und Lackiererhandwerk}

Mit der Ersten Verordnung über zwingende Arbeitsbedingungen wurden im Maler- und Lackiererhandwerk zum 1. Dezember 2003 erstmalig branchenweite Mindestlöhne durchgesetzt und durch die Zweite bis Fünfte Verordnung mit nur wenigen kurzen Unterbrechungen fortgeführt. Durch die Mindestlöhne erfasst werden Betriebe und selbständige Betriebsabteilungen, in denen mehr als $50 \%$ der Gesamtarbeitszeit der Beschäftigten für Tätigkeiten des Maler- und Lackiererhandwerks erbracht werden. Der persönliche Geltungsbereich des Mindestlohns entspricht den gewerblichen Beschäftigten, die eine versicherungspflichtige Tätigkeit ausüben. Eingeschlossen sind ferner geringfügig Beschäftigte und Aushilfskräfte, auch wenn diese keine Rentenversicherungsbeiträge leisten. Leiharbeiterinnen und Leiharbeiter, die Malerarbeiten ausführen, werden ebenfalls vom Mindestlohn erfasst. Fahrzeug- und Metalllackierer, die in stationären Werkstätten tätig sind, jugendliche Arbeitnehmerinnen und Arbeitnehmer (bis 18 Jahre) ohne abgeschlossene Berufsausbildung sowie gewerbliches Reinigungspersonal und anderes gewerbefremdes Hilfspersonal werden dagegen nicht erfasst. Kaufmännische oder technische Angestellte zählen nicht zu den gewerblichen Arbeitnehmerinnen und Arbeitnehmern und sind daher ebenfalls ausgeschlossen.

Die Mindestlöhne sind sowohl zwischen Ostdeutschland (exklusive Berlin) und Westdeutschland (inklusive Berlin) sowie zwischen gelernten und ungelernten Beschäftigten differenziert. Bei der Anwendung gilt das Arbeitsortprinzip (Baustelle), so dass zum Beispiel bei einem Einsatz ei- nes ostdeutschen Anbieters auf einer westdeutschen Baustelle der West- Mindestlohn relevant ist. Als ungelernte Beschäftigte werden all jene bezeichnet, die unter Aufsicht und Anleitung arbeiten und einfache Hilfstätigkeiten ausführen. Gelernte Beschäftigte sind Arbeitnehmerinnen oder Arbeitnehmer, die einschlägige handwerkliche Tätigkeiten ausführen. Außerdem wird bei Beschäftigten, die über den Gesellenbrief im Maler- und Lackiererhandwerk oder über einen vergleichbaren, staatlich anerkannten anderen Abschluss aus dem Ausland verfügen, vorausgesetzt, dass sie Tätigkeiten im Sinne der obengenannten Definition ausführen. Zur Einstufung als gelernte Arbeitnehmerin oder gelernter Arbeitnehmer ist also entweder ein Ausbildungsabschluss oder eine einschlägige berufliche Tätigkeit hinreichend.

Die Abb. 2 und 3 zeigen die Entwicklung der Mindestlöhne seit ihrer Einführung zum 1.12.2003 in den beiden Regionen. Im zeitlichen Verlauf lässt sich erkennen, dass die Höhe der Mindestlöhne bis zur Dritten Verordnung über zwingende Arbeitsbedingungen im Maler- und Lackiererhandwerk vom 31.08.2005, die bis zum 31.03.2008 galt, fast unverändert blieb.

Die Vierte Verordnung brachte Erhöhungen zwischen 20 und 35 Cent pro Stunde.

Mit der Fünften Rechtsverordnung über zwingende Arbeitsbedingungen im Maler- und Lackiererhandwerk vom 21. Oktober 2009 wurde der westdeutsche Mindestlohn für ungelernte Kräfte von 8,05 Euro auf 9,50 Euro erhöht. In Ostdeutschland entfiel die Differenzierung in zwei Stufen. Der ostdeutsche Mindestlohn für ungelernte Beschäftigte wurde westdeutsche Niveau angehoben. Der Mindestlohn für gelernte Arbeitskräfte wurde geringfügig auf dieses $\mathrm{Ni}$ veau gesenkt. Dadurch ergaben sich für ungelernte Arbeitskräfte Steigerungen des Mindestlohns um $18 \%$ im Westen und um $27 \%$ im Osten.

Neben dem Mindestlohn existieren Lohntarifverträge zwischen dem Hauptverband Farbe, Gestaltung, Bautenschutz, dem Bundesinnungsverband des deutschen Maler- 
Abb. 3 Höhe des Mindestlohns im Maler- und Lackiererhandwerk Ostdeutschland. Quelle: Tarifvertrag Mindestlohn für das Maler- und Lackiererhandwerk in Deutschland (2003-2011)

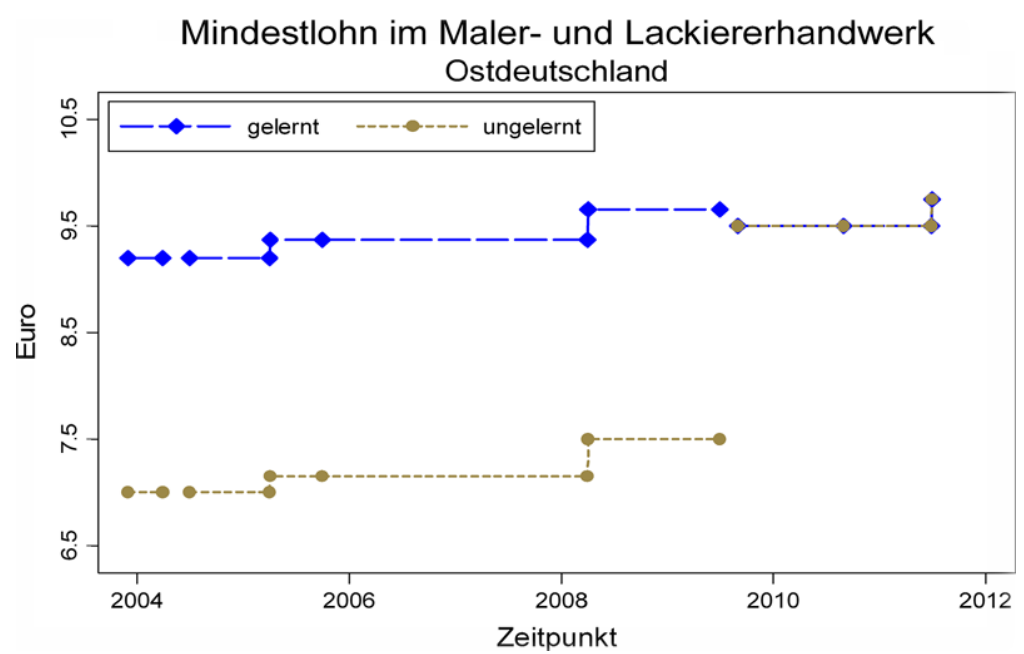

und Lackiererhandwerks und dem Bundesvorstand Industriegewerkschaft Bauen-Agrar-Umwelt. Nach einer aktuellen Umfrage sind in beiden Regionen ungefähr $60 \%$ der Unternehmen im Maler- und Lackiererhandwerk tarifgebunden (IAW 2011a). Viele der nicht tarifgebundenen Unternehmen wenden den Tarifvertrag allerdings freiwillig an, so dass die faktische Reichweite der Tarifverträge noch höher ist.

\section{3 Ökonometrisches Modell und Datengrundlagen}

Um den kausalen Effekt des Mindestlohns auf die Löhne und die Beschäftigung zu schätzen, nutzen wir die Einführung der Mindestlöhne im Jahr 2003 als natürliches Experiment und wenden ein Differenz-von-Differenzen-Verfahren (DvD) an. Der geschätzte Effekt ist damit die Differenz der zeitlichen Entwicklung der Ergebnisvariablen zwischen der Gruppe der Betroffenen und einer Kontrollgruppe. Die Kontrollgruppe besteht in unserer Anwendung aus Beschäftigten oder Betrieben in ähnlichen Branchen, die nicht in den betrieblichen Geltungsbereich der Mindestlöhne im Malerund Lackiererhandwerk fallen und auch von keinem anderen branchenspezifischen Mindestlohn erfasst werden. Hierdurch kann unter bestimmten Annahmen, die im Folgenden diskutiert werden, der durchschnittliche Treatment- Effekt auf die Personen, die dem Treatment ausgesetzt sind (Average Treatment Effect on the Treated), als Politik-Parameter geschätzt werden (Lechner 2011).

Aufgrund von Informationen aus der Verdienststrukturerhebung zur Anwendung von Tarifverträgen wird in der ökonometrischen Analyse ausschließlich die Wirtschaftsklasse 45.44.1 (Maler- und Lackierergewerbe) nach WZ 03 als Treatmentbranche verwendet (IAW 2011a: 36). Die Kontrollgruppe wurde aus den Wirtschaftsgruppen 45.3 (Bauinstallation) und 45.4 (sonstiges Ausbaugewerbe) der WZ03 ausgewählt und umfasst die Wirtschaftsunterklassen Klempnerei, Gas-, Wasser-, Heizungs- und Lüftungsinstallation
(45.33.0), Bautischlerei und -schlosserei (45.42.0), Parkettlegerei (45.43.1) und Glasergewerbe (45.44.2), da in diesen Branchen über den gesamten Untersuchungszeitraum kein Mindestlohn galt. ${ }^{2}$

Als Datengrundlage der weiteren Analysen dienen Stichproben von je 4.000 Betrieben der Treatment- und Kontrollbranchen, die für das Basisjahr 2008 aus dem Betriebshistorik-Panel ${ }^{3}$ gezogen wurden. Um auch Betriebe $\mathrm{zu}$ berücksichtigen, die im Untersuchungszeitraum am Markt vertreten waren, aber vor 2008 ausgetreten sind, wurde die Stichprobe wiederholt aufgefrischt. Auf diese Weise werden auch Betriebe erfasst, die aus dem Maler- und Lackiererhandwerk in eine andere Branche gewechselt haben. Eine Schichtung nach Ost- und Westdeutschland sowie Betriebsgrößenklassen ermöglichte eine überproportionale Berücksichtigung von ostdeutschen und großen Betrieben. Betriebe ohne Beschäftigte sind im Betriebshistorik-Panel nicht enthalten.

Für alle Beschäftigten, die in den Betrieben der Stichprobe beschäftigt waren, wurden die Integrierten Erwerbs-

\footnotetext{
${ }^{2}$ Die Auswahl der Kontrollgruppen folgte den nachfolgend angeführten Kriterien: Erstens sollten wegen der ähnlichen Entwicklung der Nachfrage nur benachbarte Branchen des Baugewerbes berücksichtigt werden. Zweitens musste ein eindeutige Zuordnung zu den Gruppen „Kontrollbranchen mit Mindestlohn“ und „Kontrollbranchen ohne Mindestlohn“ vorgenommen werden können. In einigen Kontrollbranchen wie dem Elektrohandwerk wurde ein Mindestlohn eingeführt oder abgeschafft. Andere Branchen waren aus nach den Informationen, die zum Zeitpunkt der Stichprobenziehung vorlagen, nur teilweise von einem Mindestlohn-Tarifvertrag betroffen. Zur Einordnung wurde auf Informationen aus Rahmentarifverträgen, auf Informationsmaterial der Verbände und andere Rechercheergebnisse zurückgegriffen. Die Betrachtung des Verlaufs der Ergebnisvariablen zur Prüfung von Common Trends wurde anschließend nicht nur für die Kontrollgruppen insgesamt, sondern auch für die einzelne Kontrollbranchen durchgeführt. Dabei ergab sich keine Evidenz, die zu einem Ausschluss bestimmter Kontrollbranchen geführt hätte.

${ }^{3}$ Hethey-Maier und Seth (2010) geben eine detaillierte Beschreibung des BHP.
} 
biographien $^{4}$ (IEB) zugespielt. Neben Merkmalen auf Betriebsebene stehen uns deshalb für jeden Beschäftigten eines Stichprobenbetriebs die Erwerbsbiographie, das Einkommen und andere individuelle soziodemographische Charakteristika für einen Zeitraum zwischen 1995 und 2009 zur Verfügung. Dies gilt allerdings nicht für Leiharbeiter, da diese nicht bestimmten Branchen zugewiesen werden können.

Für die vorliegende Studie ist es problematisch, dass das Einkommen in den IEB als Tagesbruttoentgelt vorliegt und keine Information über die gearbeitete Stundenanzahl enthalten ist. Der Mindestlohn stellt jedoch eine untere Schwelle für den Stundenlohn und nicht für das Tagesentgelt dar. Daher wird die Zahl der gearbeiteten Stunden auf Basis des Mikrozensus geschätzt und das Tagesentgelt durch diese Größe dividiert. Hierfür wird die durchschnittliche übliche Wochenarbeitszeit differenziert nach Jahr und Berufsbildung (alle Bildungsniveaus, Bildungsniveau unbekannt/ohne Lehre sowie abgeschlossene Lehre) berechnet und unseren Daten zugespielt. Zusätzlich wird danach unterschieden, ob die Berufsgruppe dem persönlichen Geltungsbereich angehört oder nicht. Es werden nur Stundenlöhne für Vollzeitbeschäftigte berechnet, da andere Arbeitszeitkategorien aufgrund der Fallzahl keine ausreichend feine Gliederung zulassen. ${ }^{5}$

Beschäftigungsgruppen wie nicht-gewerbliche Beschäftigte, Auszubildende oder Praktikanten, für die der Mindestlohn nicht gilt, werden aus der Analyse ausgeschlossen. Außerdem werden Meister und Poliere sowie Beschäftigte mit einem Hochschulabschluss nicht berücksichtigt, da diese mit großer Wahrscheinlichkeit nicht direkt vom Mindestlohn betroffen sind. Diese Herangehensweise soll verhindern, dass nicht vom Mindestlohn betroffene Personen der Treatmentgruppe zugewiesen werden, denn dies könnte die Schätzergebnisse abschwächen. ${ }^{6}$ Um mögliche Substitutionseffekte zu minimieren, werden außerdem Maler und Lackierer aus der Kontrollgruppe ausgeschlossen. Beobachtungen mit Stundenlöhnen von weniger als 3 Euro oder mehr als 50 Euro resultieren wahrscheinlich aus Messfehlern und werden ebenfalls ausgeschlossen.

\footnotetext{
${ }^{4}$ Eine detaillierte Beschreibung einer Stichprobe der IEB, der IEB-S, befindet sich in Oberschachtsiek et al. (2009).

${ }^{5}$ Um die Validität der auf diese Weise berechneten Stundenlöhne zu überprüfen, ziehen wir eine weitere Datenquelle, die Verdienststrukturerhebung (VSE), heran. Die Lohnverteilungen beider Quellen ähneln sich stark. Die beiden Datenquellen sind allerdings nicht perfekt vergleichbar, da beispielsweise Unternehmen mit unter 10 Beschäftigten in der VSE nicht enthalten sind.

${ }^{6}$ Von der Eingrenzung auf wahrscheinlich betroffene Beschäftigte auf Basis des vor der Einführung beobachteten Lohns wird hier abgesehen, da hierfür ein entsprechender Schwellenwert für die Kontrollbranchen geschätzt werden müsste. Außerdem könnten Beschäftigte, die nach der Mindestlohneinführung eingestellt wurden, nicht berücksichtigt werden, und mögliche indirekte Effekte auf höhere Lohngruppen würden nicht eingefangen.
}

Bei der Evaluation der Mindestlöhne im Maler- und Lackiererhandwerk stellt sich die zusätzliche Herausforderung, Gruppen abzugrenzen, die vom Mindestlohn für gelernte und ungelernte Beschäftigte betroffen sind. Da für die Einstufung in den Geltungsbereich des Mindestlohns sowohl ein einschlägiger Ausbildungsabschluss als auch eine einschlägige berufliche Tätigkeit hinreichend ist, wird hier zur Abgrenzung die IEB-Variable „Stellung im Beruf und Arbeitszeit" genutzt, mit der zwischen Nicht-Facharbeitern und Facharbeitern unterschieden werden kann. Die Abgrenzung erfolgt dabei über das Kennzeichen des Rentenversicherungsträgers. $^{7}$

Die zentrale Annahme des DvD-Schätzers ist die Annahme eines gemeinsamen Trends, das heißt, dass die Entwicklung der Ergebnisvariablen für die Treatment- und Kontrollgruppe bei Abwesenheit des Treatments gleich gewesen wäre. ${ }^{8}$ Der Plausibilität dieser Annahme muss besondere Bedeutung beigemessen werden. Werden, wie das hier der Fall ist, Kontroll- und Treatmentgruppe aus unterschiedlichen Branchen gezogen, ist beispielsweise $\mathrm{zu}$ beachten, dass diese unterschiedlichen makroökonomischen Schocks ausgeliefert sein könnten. Dies könnte eine Verzerrung des Schätzers in unbekannter Richtung zur Folge haben. Da sich allerdings sowohl Treatment- als auch Kontrollgruppe aus Branchen des Baugewerbes zusammensetzt, ist die wirtschaftliche Entwicklung beider Gruppen stark von der Entwicklung des Bausektors geprägt, was einen gemeinsamen Trend der Ergebnisvariablen begünstigen dürfte.

$\mathrm{Ob}$ die Kontrollgruppe valide ist, kann formal nicht getestet werden, da das kontrafaktische Ergebnis, das eingetreten wäre, wenn es kein Treatment gegeben hätte, naturgemäß nie beobachtet werden kann. Einen Hinweis auf die Plausibilität der Annahme können insbesondere folgende Analysen liefern: ein Vergleich der Zusammensetzung von Kontroll- und Treatmentgruppe mit Hinblick auf Charakteristika, die einen abweichenden Trend verursachen könnten sowie ein Vergleich der beobachteten Entwicklungen der Ergebnisvariablen vor dem Treatment. ${ }^{9}$

Zur Feststellung von Unterschieden in der Zusammensetzung werden $t$-Tests auf Gleichheit der Mittelwerte von ins-

\footnotetext{
${ }^{7}$ Eine Alternative stellt das IEB-Merkmal „Ausbildung“ dar, welches allerdings nicht den Ausbildungsberuf enthält und damit zu einer ungenaueren Abgrenzung führen könnte. Da allerdings persönliche Merkmale wie Ausbildung oder die Stellung im Beruf aufgrund fehlender Relevanz für die Sozialversicherungsträger mit größerer Vorsicht zu benutzen sind als etwa Beschäftigungszeiten (Fitzenberger et al. 2005), wurden zusätzliche Robustheitsanalysen durchgeführt, in denen das IEB-Merkmal „Ausbildung“ verwendet wurde.

${ }^{8}$ Weitere Annahmen sind, dass das Treatment nicht antizipiert wird und somit zum ersten Messzeitpunkt keine Wirkungen auf die Ergebnisvariablen hat. Zudem muss sichergestellt sein, dass die Kontrollgruppe nicht durch das Treatment beeinflusst wird.

${ }^{9}$ Eine weitere Möglichkeit, die Annahme eines gemeinsamen Trends zu prüfen, sind Placebo-Experimente. Siehe dazu Abschn. 4 und 5.
} 
gesamt 79 individuellen, betrieblichen und regionalen Charakteristika durchgeführt. In den meisten Fällen wird die Nullhypothese der Gleichheit der Mittelwerte zwar zurückgewiesen, die Größenordnung fast aller Unterschiede ist allerdings sehr gering (siehe die Tab. 5 im Anhang). Ein gröBerer Unterschied ergibt sich beim Anteil geringfügig Beschäftigter und Beschäftigung als Nebentätigkeit, der in den Kontrollbranchen höher ist. Entsprechend ist auch der Anteil der Beschäftigten mit einfachen manuellen Berufen höher als in der Treatmentgruppe. Ferner sind die Personen in der Kontrollgruppe häufiger als Angestellte klassifiziert und dafür seltener als Facharbeiter. Dies könnte ein Grund dafür sein, dass in der Kontrollgruppe mehr Personen enthalten sind, die einen Hochschulabschluss haben. Ein größerer Anteil der Beschäftigten in der Kontrollgruppe arbeitet außerdem in Großbetrieben mit mehr als 100 Beschäftigten, während der Anteil mittelgroßer Betriebe (11 bis $100 \mathrm{Be}-$ schäftigte) in der Treatmentgruppe größer ist.

Im Bericht der Evaluation werden außerdem Ergebnisse für weitere Kontrollgruppen aufgeführt, die als Robustheitsprüfungen der hier vorgestellten Ergebnisse verwendet werden können:

- Beschäftigte in vergleichbaren Branchen, für die seit 1997 ein Mindestlohn galt und für die deshalb im Jahr 2003 keine Änderung eintrat. Diese vergleichbaren Branchen sind die Wirtschaftsunterklassen Hoch- und Tiefbau, ohne ausgeprägten Schwerpunkt (45.21.1), Hochbau ohne Fertigteilbau (45.21.2), Errichtung von Fertigteilbauten aus Beton im Hochbau aus selbst hergestellten Fertigteilen (45.21.3), Errichtung von Fertigteilbauten aus Beton im Hochbau aus fremd bezogenen Fertigteilen (45.21.4), Errichtung von Fertigteilbauten aus Holz und Kunststoffen im Hochbau aus fremd bezogenen Fertigteilen (45.21.5) und Zimmerei und Ingenieurholzbau (45.22.3).

- Maler und Lackierer, die in anderen Branchen des Bausektors beschäftigt sind. Hierbei wurden aus Fallzahlengründen nur solche Branchen einbezogen, die einen Mindestlohn haben.

- Beschäftigte mit etwas höherem Lohn als der Mindestlohn wie bei König und Möller (2008). In unserer Anwendung besteht die Kontrollgruppe aus Personen, die vor Einführung des Mindestlohns einen Stundenlohn bezogen, der mindestens so hoch war wie der Mindestlohn, aber aus Gründer der Vergleichbarkeit höchstens $15 \%$ darüber lag.

In den Analysen zur Plausibilität der gemeinsamen Trends stellte sich die Kontrollgruppe aus Branchen ohne Mindestlohn tendenziell als besser geeignet dar als die Kontrollgruppen aus Mindestlohn-Branchen. Für die Verwendung der Kontrollgruppe mit Personen aus höheren Lohngruppen spricht insbesondere, dass diese der gleichen Branche angehören. Damit ist die Annahme eines gemeinsamen Trends nicht durch die Heterogenität zwischen unterschiedlichen Branchen und Tätigkeiten gefährdet. Allerdings ist die Annahme, dass die Kontrollgruppe nicht vom Mindestlohn beeinflusst wird, problematisch. Theoretische Gründe sprechen dafür, dass der Mindestlohn auch auf höhere Bereiche der Lohnverteilung Einfluss hat, die nicht direkt vom Mindestlohn betroffen werden. Die Ergebnisse von Rattenhuber (2011) bestätigen dies für das Bauhauptgewerbe. Qualitative Ergebnisse liefern Anhaltspunkte dafür, dass dies auch im Maler- und Lackiererhandwerk zutreffen könnte (IAW 2011a). Dies würde dazu führen, dass der Effekt des Mindestlohns in der quantitativen Analyse unterschätzt wird. ${ }^{10}$

Auch vom Mindestlohn nicht betroffene Betriebe könnten von Substitutionseffekten oder anderen spillover-Effekten betroffen sein. Da wir allerdings Maler und Lackierer aus anderen Branchen nicht in der Kontrollgruppe berücksichtigen, sind Substitutionseffekte hier als weniger problematisch anzusehen.

Aufgrund dieser Gesichtspunkte führt die Wahl der Kontrollgruppe aus Betrieben ohne Mindestlohn unserer Meinung nach insgesamt am ehesten zu validen Ergebnissen. ${ }^{11}$ Die Ergebnisse der Robustheitsanalysen auf Basis der anderen Kontrollgruppen werden im Text diskutiert.

\section{Lohnwirkungen}

Zunächst werden die Daten über die Stundenentgelte aus der Beschäftigtenstatistik bzw. dem Mikrozensus verwendet, um die Lohnverteilung und die Eingriffsintensität des Mindestlohns deskriptiv zu analysieren. Die Verteilungen werden mit Hilfe von Kerndichteschätzungen dargestellt. Dabei werden die Jahre 2001 und 2005 als Vergleichsjahre vor und nach Einführung des Mindestlohns betrachtet. Für die Analysen der Löhne werden nur Vollzeitkräfte herangezogen, weil sich nur für diese der Stundenlohn berechnen lässt.

Abbildung 4 zeigt, dass sich die Lohnverteilung für die gelernten Kräfte in Ostdeutschland deutlich verändert hat.

\footnotetext{
${ }^{10}$ Ein weiterer Nachteil der Kontrollgruppe innerhalb des Maler- und Lackiererhandwerks ist die relativ kleine Anzahl an Beobachtungen, die nur durch die Anhebung der Lohnobergrenze der einbezogenen Personen erhöht werden kann, was allerdings auf Kosten der Vergleichbarkeit der beiden Gruppen geschehen würde. Effekte des Mindestlohns auf ungelernte Beschäftigte konnten deshalb auf der Basis dieses Kontrollgruppenansatzes nicht analysiert werden.

${ }^{11}$ In einem weiteren Verfahren wird die Kontrollgruppe durch solche Betriebe gebildet, die an einen Lohntarifvertrag gebunden sind, der mindestens so hohe Entgelte vorsieht wie der Mindestlohn. Diese Betriebe sollten durch den Mindestlohn nicht direkt beeinflusst werden. Hier ergibt sich allerdings ein Problem der Abgrenzung, da tarifliche Normen auch freiwillig angewendet werden können. Aus diesem Grund wird im Folgenden auf die entsprechenden Ergebnisse nicht Bezug genommen.
} 

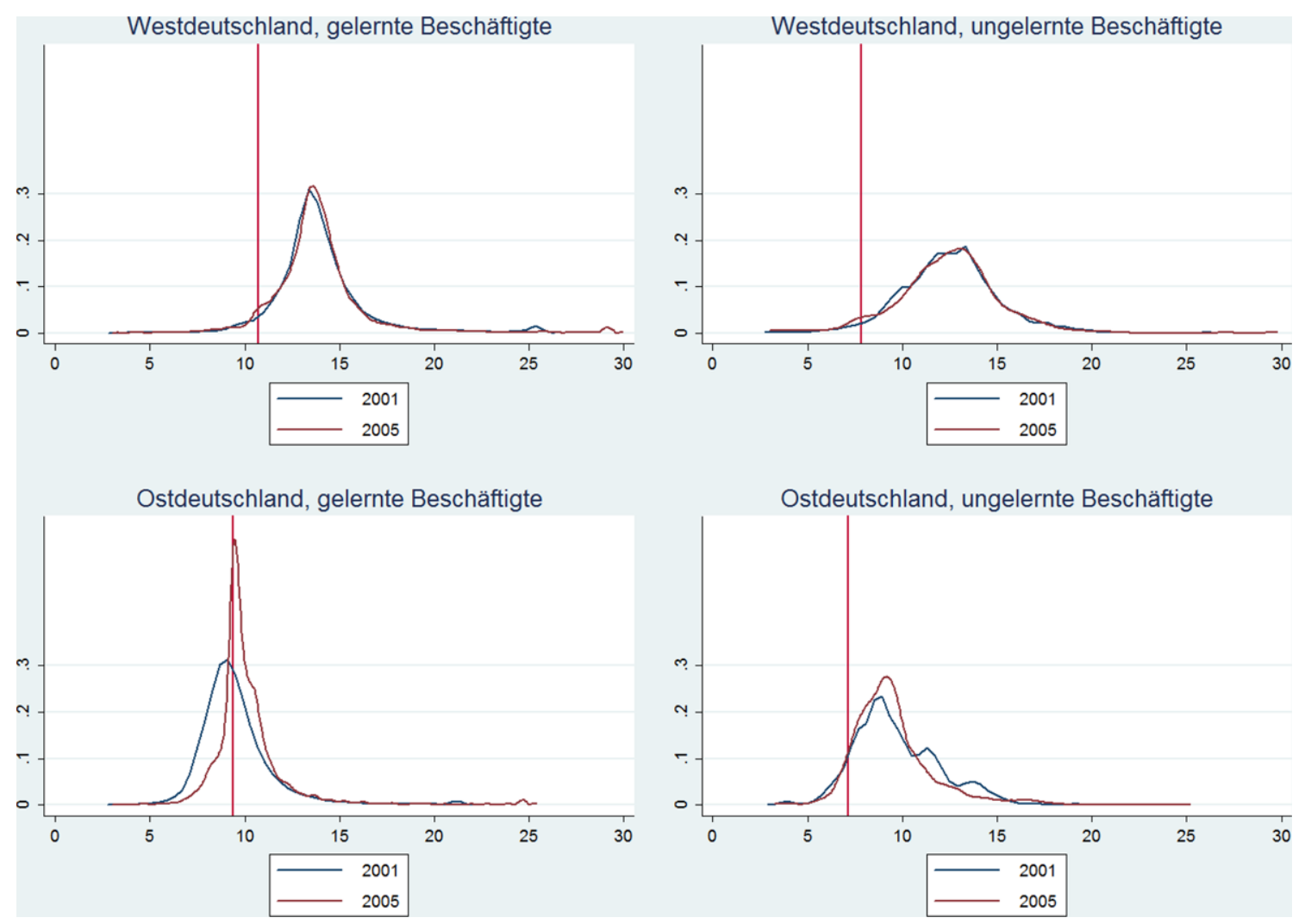

Abb. 4 Verteilung der Stundenlöhne nach IEB/Mikrozensus, 2001-2005. Vertikale Linien geben den Mindestlohn in der Höhe zum Einführungszeitpunkt 2003 an. Quelle: Integrierte Erwerbsbiographien (IEB)/Mikrozensus

Im Jahr 2001 ist die Verteilung symmetrisch; ihr Modus liegt deutlich unterhalb des künftigen Mindestlohns. Bis zum Jahr 2005 hat sich der Modus nach rechts verschoben und liegt nun ungefähr auf der Höhe des Mindestlohns. Die Verteilung ist unterhalb des Mindestlohns deutlich gestaucht. Auch bei Löhnen oberhalb des Mindestlohns (im Bereich ungefähr zwischen 9,50 Euro und 12,00 Euro) lassen sich Änderungen feststellen. Die Häufigkeit von Löhnen in dieser Höhe hat sich deutlich erhöht. Dies könnte ein Anzeichen für indirekte Effekte der Mindestlöhne auf die gesamte Lohnverteilung sein.

Bei den ungelernten Kräften in Ostdeutschland verändern sich Form und Lage der Lohnverteilung zwischen 2001 und 2005 dagegen in weitaus geringerem Maße. Bereits vor Einführung der Mindestlöhne bezieht nur ein relativ kleiner Teil der Beschäftigten weniger als den künftigen Mindestlohn. Eine geringfügige Stauchung der Lohnverteilung unterhalb des Mindestlohns ist jedoch zu erkennen. Zugleich ist festzustellen, dass Löhne deutlich oberhalb des Mindestlohns relativ seltener zu finden sind, so dass sich eine Verminderung der Streuung und damit eine Lohnkompression ergeben. In Westdeutschland blieb die Lohnverteilung zwischen 2001 und 2005 praktisch unverändert. Allenfalls eine minimale Rechtsverschiebung der Löhne der ungelernten Kräfte lässt sich erkennen.
In Übereinstimmung mit den grafisch dargestellten Lohnverteilungen ist die Eingriffsintensität der Mindestlöhne bei den gelernten Kräften in Ostdeutschland weitaus am höchsten (Tab. 1). So beträgt der Kaitz-Index, also das Verhältnis von Mindestlohn zum Medianlohn, in der ex-ante-Messung vor Einführung des Mindestlohns fast $100 \%$. Fast die Hälfte der Beschäftigten ist also direkt vom Mindestlohn betroffen, indem ihr Lohn unter dem Mindestlohn oder auf derselben Höhe wie der Mindestlohn liegt. ${ }^{12}$

Bis zum Jahr 2006 geht der Kaitz-Index in der Gruppe der gelernten Arbeitskräfte in Ostdeutschland um einige Prozentpunkte zurück. Dies ist auf die Rechtsverschiebung der Lohnverteilung bei den gelernten Kräften in Ostdeutschland zurückzuführen. Der Rückgang des Kaitz-Index fällt

\footnotetext{
${ }^{12}$ Die Ergebnisse für gelernte Beschäftigte wurden mit der Verdienststrukturerhebung (VSE) repliziert (IAW 2011a). Diese Datenquelle hat den Vorteil, dass sie exakte Informationen über die Stundenlöhne enthält. Die Werte der VSE für den Kaitz-Index und den Anteil der Betroffenen sind für Ostdeutschland um weniger Prozentpunkte höher, während sie für Westdeutschland etwas niedriger sind. Dabei ist allerdings eine leicht unterschiedliche Gesamtpopulation in den Datenquellen zu berücksichtigen. Die Veränderung der Werte über die Zeit ist in beiden Datenquellen sehr ähnlich. Das spricht dafür, dass durch die Imputation der gearbeiteten Stunden keine wesentlichen Verzerrungen in der Lohnverteilung entstehen.
} 
Tab. 1 Kennzahlen zur Eingriffsintensität des Mindestlohns

\begin{tabular}{|c|c|c|c|c|}
\hline & \multicolumn{2}{|c|}{ Ostdeutschland } & \multicolumn{2}{|c|}{ Westdeutschland } \\
\hline & Gelernt & Ungelernt & Gelernt & Ungelernt \\
\hline \multicolumn{5}{|l|}{ Ex ante-Maße für 2003} \\
\hline Kaitz Index ex ante & 97,6 & 73,3 & 76,1 & 59,5 \\
\hline Anteil direkt Betroffener & 48,9 & 7,8 & 4,8 & 4,2 \\
\hline Anteil am Mindestlohn & 9,8 & 2,0 & 1,3 & 1,0 \\
\hline \multicolumn{5}{|c|}{ Kontemporäre Maße für 2006} \\
\hline Kaitz Index ex ante & 93,6 & 75,9 & 78,3 & 61,8 \\
\hline Anteil direkt Betroffener & 33,9 & 6,8 & 6,3 & 4,3 \\
\hline Anteil am Mindestlohn & 18,7 & 2,1 & 1,9 & 1,0 \\
\hline
\end{tabular}

Anmerkungen: Alle Zahlen in Prozent. Anteil direkt Betroffener: Anteil der Beschäftigten, deren Lohn maximal das 1,02-fache des Mindestlohn beträgt, an allen Beschäftigten im Geltungsbereich. Anteil am Mindestlohn: Anteil der Beschäftigten, deren Lohn zwischen dem 0,98-fachen und dem 1,02-fachen des Mindestlohns beträgt. Quelle: Integrierte Erwerbsbiographie, Mikrozensus

deshalb nicht stärker aus, weil der Mindestlohn zugleich im März 2005 um 33 Cent erhöht wurde.

Deutlich sinkt von 2003 bis 2006 der Anteil der gelernten Arbeitskräfte in Ostdeutschland, die unter oder direkt am Mindestlohn liegen; dieser beträgt 2006 nur noch ein Drittel der Beschäftigten. Damit ergeben sich erste Hinweise für Lohnwirkungen. Etwa 15 Prozent der gelernten Beschäftigten in Ostdeutschland verdienen im Jahr 2006 weniger als den Mindestlohn für Gelernte.

Bei den ungelernten Kräften in Ostdeutschland ist die Eingriffsintensität allen verfügbaren Maßen zufolge erheblich geringer als bei den gelernten. Der Anteil der direkt vom Mindestlohn Betroffenen liegt vor Einführung des Mindestlohns bei $8 \%$ und sinkt danach auf $7 \%$. Die Zunahme des Kaitz-Index von $73 \%$ auf $76 \%$ liegt an der Erhöhung des Mindestlohns bei kaum veränderter Lohnverteilung.

Auch in Westdeutschland ist die Eingriffsintensität bei den gelernten Kräften mit einem Kaitz-Index von $76 \%$ gröBer als bei den Ungelernten (60\%). Der Kaitz-Index steigt bis zum Jahr 2006 in beiden Gruppen leicht an. Der Anteil der direkt Betroffenen liegt im Ausgangspunkt bei $4 \%$ (ungelernte Kräfte) bzw. $6 \%$ (gelernte). Damit hat der Mindestlohn nur für sehr wenige Beschäftigte in Westdeutschland eine unmittelbare Bedeutung.

Die Abgrenzung zwischen gelernten und ungelernten Kräften in den Daten ist mit Schwierigkeiten verbunden, wie in Abschn. 3 ausgeführt wurde. Daher ist zu fragen, ob das Ergebnis, dass mit $15 \%$ ein relativ hoher Anteil der gelernten Kräfte in Ostdeutschland im Jahr 2006 weniger Lohn erhält als der für diese Gruppe vorgesehene Mindestlohn, tatsächlich auf die unzureichende Anwendung des Mindestlohns für gelernte Arbeitskräfte seitens der Betriebe zurückgeht oder auf eine falsche Zuordnung ungelernter Arbeitskräfte zu den gelernten in den Datenquellen. Für die erste Erklärung spricht, dass in Expertengesprächen, die für die- ses Projekt durchgeführt wurden, Hinweise auf Nichteinhaltung gefunden wurden. Es wurde außerdem angegeben, dass es auch in der Praxis Probleme gibt, Beschäftigte in gelernt und ungelernt zu differenzieren (IAW 2011a).

Zusammenfassend zeichnen sich hinsichtlich der Eingriffsintensität deutliche Unterschiede zwischen Ost- und Westdeutschland ab. In Westdeutschland ist die Eingriffsintensität gering. Gestalt und Lage der Lohnverteilung ändern sich mit der Einführung des Mindestlohns kaum. In Ostdeutschland ist die Eingriffsintensität ex ante erheblich. Dies betrifft vor allem die gelernten Arbeitskräfte, wobei allerdings die Zuordnung zu den Bereichen gelernte und ungelernte Arbeitskräfte problematisch ist.

Den Verlauf des durchschnittlichen Stundenlohns über den Zeitraum von zwischen 1995 und 2008 zeigt Abb. 5. Die vertikale Linie kennzeichnet das Jahr der Einführung des Mindestlohns. Ein Lohnwachstum ist in der Teilnehmergruppe (d.h. im Maler- und Lackiererhandwerk) vor allem in der Gruppe der gelernten Kräfte in Ostdeutschland festzustellen. Nur eine geringe Zunahme findet sich dagegen bei den ungelernten Kräften.

Abbildung 5 führt zugleich die Lohnentwicklung in der Kontrollgruppe auf. Damit kann die Validität der Annahme eines gemeinsamen Trends beurteilt werden; relevant ist nur die Entwicklung im grau unterlegten Zeitraum vor dem Treatment. ${ }^{13}$ Bei den gelernten Kräften in Ostdeutschland verläuft die Lohnentwicklung in beiden Gruppen von 1999 bis 2002 parallel; davor ist ein leichter Rückgang in den Kontrollbranchen, nicht aber in der Treatment-Branche festzustellen. Ein ähnliches Bild ergibt sich in Westdeutschland, wobei hier ein Niveauunterschied hinzukommt. Bei den ungelernten Kräften sind gleich verlaufende Trends in

\footnotetext{
${ }^{13}$ Der Zeitraum danach sowie der Datenpunkt im Jahr des Treatments können dagegen bereits vom Mindestlohn beeinflusst sein.
} 
Abb. 5 Entwicklung der Durchschnittslöhne. Quelle: IEB/Mikrozensus

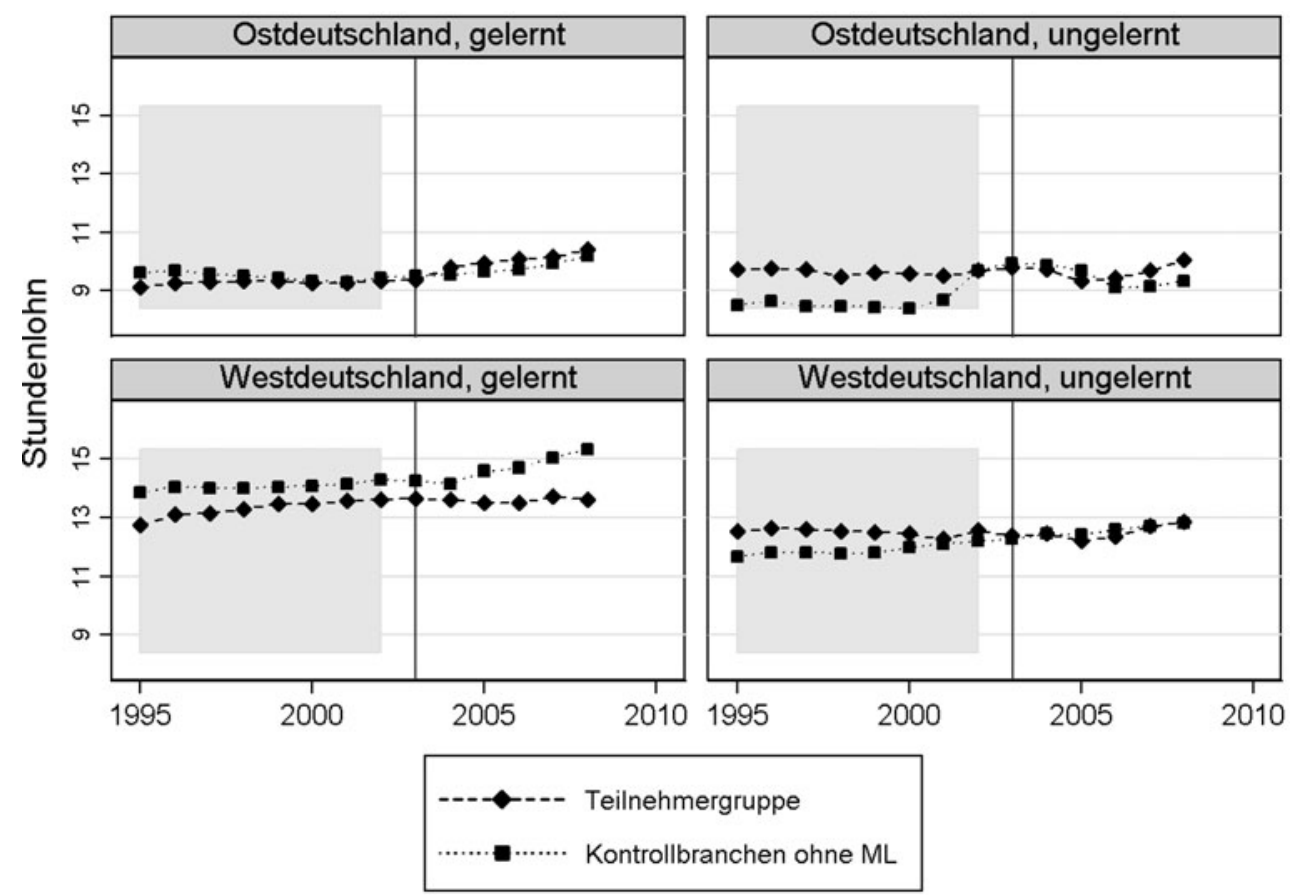

weitaus geringerem Maße sichtbar. Hier zeigt sich insbesondere in Ostdeutschland von 2001 bis 2003 ein Anstieg der Löhne in der Kontrollgruppe, der sich in der TreatmentGruppe nicht feststellen lässt. Dies wirft Zweifel an der Validität der entsprechenden Ergebnisse mit dem Differenz-vonDifferenzen-Verfahren auf.

In den Kausalanalysen werden in einem konditionalen DvD-Ansatz Lohneffekte in einer linearen Regression mit dem Niveau des logarithmierten durchschnittlichen Stundenentgelts als abhängiger Variable geschätzt. Um potentielle Antizipationswirkungen oder Wirkungsverzögerungen zu berücksichtigen, wurde als ex-ante Messzeitpunkt der 1 . September 2002 gewählt. ${ }^{14}$ Alternative Zeitpunkte für die zweite Messung sind jeweils der 1. September der Jahre 2004 und 2005. Damit lassen sich kürzer- und längerfristige Lohnwirkungen darstellen.

Als unabhängige Variablen werden in allen Analysen individuelle Merkmale (zum Beispiel Ausbildung, Altersklassen, Arbeitserfahrung oder Nationalität) und betriebliche Charakteristika, wie die Betriebsgröße oder die $\mathrm{Zu}$ sammensetzung der Belegschaft (beispielsweise bezüglich Alter, Ausbildung, Anteil Arbeiter und Angestellter, Anteil

\footnotetext{
${ }^{14}$ Es ist außerdem zu berücksichtigen, dass in den IEB die Information über das gezahlte Entgelt als durchschnittliches Tagesentgelt vorliegt für all die Kalendertage in einem bestimmten Jahr, die eine Beschäftigungsepisode umfasst. Überlappt eine Beschäftigungsepisode zum Beispiel die Einführung eines Mindestlohns, ist das Tagesentgelt ein gewichteter Durchschnitt des Lohns vor und nach dem natürlichen Experiment, weshalb die Messzeitpunkte nicht im Jahr der Mindestlohneinführung liegen sollten.
}

Teilzeit, Vollzeit oder Geringfügigkeit), aufgenommen. Zudem wird für regionale Charakteristika wie Siedlungstyp, regionale Arbeitslosenquote sowie für das Bundesland kontrolliert. Die Mittelwerte der verwendeten Kovariate werden im Anhang getrennt nach Kontroll- und Treatmentgruppe aufgeführt.

Im Folgenden werden Teile der Ergebnisse der Differenzvon-Differenzen-Schätzungen für die Ergebnisvariable Stundenlohn präsentiert. Tabelle 2 enthält den Treatment-Effekt, der durch den Koeffizienten des Interaktionseffekts zwischen Treatmentgruppe und Periode geschätzt wird, sowie die beiden linearen Effekte der Dummy-Variablen für die Treatmentgruppe und die jeweilige ex-post Periode. Außerdem wird die Zahl der Beobachtungen und der Wert des Bestimmtheitsmaßes $R^{2}$ angegeben. Die geschätzten Effekte der Kovariate werden aus Platzgründen nicht dargestellt.

Die Ergebnisse für den Interaktionsterm zeigen nur für gelernte Beschäftigte in Ostdeutschland einen statistisch signifikanten Effekt des Mindestlohns. Die Größenordnung des Koeffizienten bedeutet, dass die Stundenlöhne bei den gelernten Kräften durch die Einführung des Mindestlohns bis 2004 um $4 \%$ und bis 2005 um $6 \%$ gestiegen sind. Für die anderen Beschäftigten-Gruppen sind die Punktschätzer fast null und der $t$-Wert weit von den üblichen kritischen Werten entfernt. Dieses Ergebnis ist konsistent mit der deskriptiven Analyse, die nur für gelernte Beschäftigte in Ostdeutschland eine signifikante Eingriffsintensität ergab.

Betrachtet man die Haupteffekte, erkennt man nur geringfügige Lohnänderungen im betrachteten Zeitraum. Für gelernte Beschäftigte in Ostdeutschland und ungelernte Beschäftigte in Westdeutschland hat die Zugehörigkeit zur 
Tab. 2 Wirkung der Einführung des Mindestlohns auf den Stundenlohn

\begin{tabular}{|c|c|c|c|c|}
\hline & \multicolumn{2}{|c|}{ Kurze Frist (2004) } & \multicolumn{2}{|c|}{ Lange Frist (2005) } \\
\hline & Gelernte & Ungelernte & Gelernte & Ungelernte \\
\hline \multicolumn{5}{|l|}{ Ostdeutschland } \\
\hline Treatmentgruppe & $0,04^{* * *}(3,62)$ & $0,03(1,14)$ & $0,03^{* * *}(3,18)$ & $0,02(0,71)$ \\
\hline Zeiteffekt & $0,01^{*}(1,97)$ & $0,02(0,95)$ & $0,01(1,17)$ & $-0,01(0,51)$ \\
\hline Treatment-Effekt & $0,04^{* * *}(5,98)$ & $0,00(0,01)$ & $0,06^{* * *}(7,36)$ & $0,03(1,02)$ \\
\hline Weitere Kovariate & $\mathrm{Ja}$ & $\mathrm{Ja}$ & $\mathrm{Ja}$ & $\mathrm{Ja}$ \\
\hline Placebo-Test, $p$-Wert & 0,50 & 0,29 & 0,54 & $0,02^{* *}$ \\
\hline$R^{2}$ & 0,25 & 0,47 & 0,25 & 0,44 \\
\hline Anzahl der Beobachtungen & 23.538 & 2.372 & 22.471 & 2.310 \\
\hline \multicolumn{5}{|l|}{ Westdeutschland } \\
\hline Treatmentgruppe & $0,01(0,67)$ & $0,04^{* * *}(2,76)$ & $0,01(1,16)$ & $0,05^{* * *}(3,24)$ \\
\hline Zeiteffekt & $-0,01^{* * *}(2,69)$ & $-0,01(0,51)$ & $0,01(0,44)$ & $-0,00(0,23)$ \\
\hline Treatment-Effekt & $0,00(0,70)$ & $-0,01(0,64)$ & $-0,02(1,30)$ & $-0,04^{*}(1,87)$ \\
\hline Weitere Kovariate & $\mathrm{Ja}$ & $\mathrm{Ja}$ & $\mathrm{Ja}$ & $\mathrm{Ja}$ \\
\hline Placebo-Test, $p$-Wert & 0,37 & 0,90 & 0,27 & $0,04^{* *}$ \\
\hline$R^{2}$ & 0,31 & 0,31 & 0,32 & 0,28 \\
\hline Anzahl der Beobachtungen & 46.063 & 6.449 & 45.463 & 6.622 \\
\hline
\end{tabular}

Anmerkungen: Absolute $t$-Statistiken basieren auf geclusterten Standardfehlern (auf Betriebsebene) und sind in Klammern unter den Koeffizienten abgetragen. Statistische Signifikanz auf dem $1 \%$-, $5 \%$ - und $10 \%$-Niveau wird durch ${ }^{* * *},{ }^{* *}$ und ${ }^{*}$ gekennzeichnet

Treatmentgruppe einen positiven Effekt. Für die beiden anderen Gruppen unterscheiden sich Treatment- und Kontrollgruppen hinsichtlich der Lohnhöhe zum Zeitpunkt der Nullmessung statistisch nicht.

Die Koeffizienten der unabhängigen Variablen (nicht aufgeführt) deuten auf plausible Effekte sowohl in der Richtung als auch in der Größenordnung hin. So steigt der Stundenlohn monoton mit dem Alter und der Betriebszugehörigkeit. Gering qualifizierte Kräfte erhalten einen niedrigeren Stundenlohn. Beschäftigte in größeren Betrieben (ab 100 Beschäftigte) erhalten einen deutlich höheren Stundenlohn. Diese Resultate entsprechen denen, die auf der Basis anderer Daten in Lohnregressionen erzielt werden. Das Bestimmtheitsmaß $R^{2}$ beträgt in den Schätzungen zwischen 0,25 und 0,47 und liegt damit ebenfalls im üblichen Rahmen.

In der jeweils drittletzten Zeile werden die Ergebnisse von Placebo-Experimenten dargestellt. Dabei wird eine kontrafaktische Mindestlohneinführung im Jahr 2001 analysiert. Die Lohnmessung verschiebt sich ebenfalls um jeweils zwei Jahre. Signifikante „Treatment“-Effekte sind ein klarer Hinweis dafür, dass die Annahme gleich verlaufender Trends nicht zutrifft. Die Tabelle enthält den $p$-Wert für den geschätzten Koeffizienten des Interaktionseffekts. Die Ergebnisse der Placebo-Tests zeigen, dass für die ungelernten Kräfte im längerfristigen Untersuchungszeitraum die Annahme vermutlich nicht erfüllt ist. Dies gilt für Ostund Westdeutschland. Hinsichtlich der Schätzungen für die gelernten Kräfte kann die Annahme gemeinsamer Trends nicht zurückgewiesen werden. Diese Ergebnisse stehen im Einklang mit der deskriptiven Analyse der Lohnentwicklung in beiden Gruppen.

Ergebnisse mit alternativen Kontrollgruppen finden teilweise auch für die gelernten Kräfte in Ostdeutschland keinen signifikanten Treatment-Effekt. Im Fall der Kontrollgruppe aus Mindestlohnbranchen zeigt das PlaceboExperiment allerdings an, dass die Annahme gemeinsamer Trends für Ostdeutschland nicht erfüllt sein dürfte, so dass die Kontrollgruppe ungeeignet ist. Bei der Kontrollgruppe mit Beschäftigten, die vor Einführung des Mindestlohns mehr als den Mindestlohn verdienten, ergibt sich kein positiver Lohneffekt. Dies ist vermutlich darauf zurückzuführen, dass die Annahme, dass die Kontrollgruppe nicht durch das Treatment beeinflusst wird, verletzt ist. Denn die deskriptive Analyse der Lohnverteilung zeigt klar eine Verschiebung der gesamten Lohnverteilung, nicht nur eine Stauchung der Verteilung unterhalb des Mindestlohns. Dies deutet darauf hin, dass spillover-Effekte existieren und die Ergebnisse, die mit dieser Kontrollgruppe erzielt wurden, nach unten verzerrt sind. Die Abwesenheit eines positiven Lohneffekts spricht daher nicht gegen die Validität der positiven Lohneffekte, die mit der Kontrollgruppe aus anderen Branchen geschätzt werden.

Sowohl die deskriptiven Analysen als auch die Schätzungen deuten also auf einen positiven Lohneffekt des Mindestlohns auf die gelernten Beschäftigten im ostdeutschen Maler- und Lackiererhandwerk hin, der in der Größenord- 
Abb. 6 Zeiträume für die Ergebnismessung bei der Weiterbeschäftigungswahrscheinlichkeit
01.09 .2003 - 30.11.2003

Beschäftigungsanpassungen in Antizipation des MLs

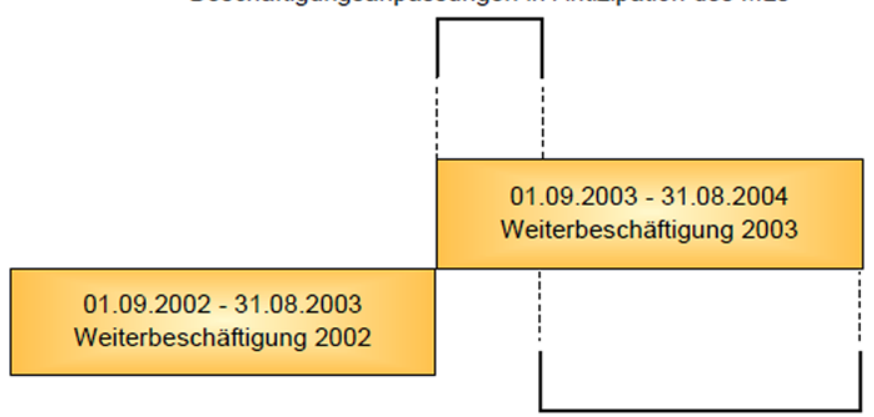

$01.12 .2003-31.08 .2004$

Beschäftigungsanpassungen als Reaktion auf $\mathrm{ML}$

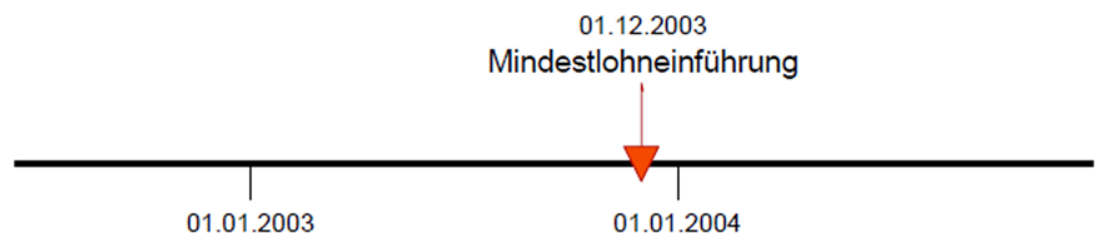

nung mit $4 \%$ bis $6 \%$ relativ stark ausgeprägt ist. Dies steht im Einklang mit den deskriptiven Befunden. In den anderen Gruppen, also bei den ungelernten Kräften und in Westdeutschland, finden sich keine signifikanten TreatmentEffekte. Für die ungelernten Kräfte erscheint die commontrends-Annahme problematisch. Auch angesichts der deskriptiven Evidenz ist hier allerdings nicht zu erwarten, dass ausgeprägte Lohnwirkungen vorhanden sein könnten. Es wäre zudem kaum plausibel, dass in Gruppen, in denen nur $4 \%$ bis $8 \%$ der Beschäftigten direkt vom Mindestlohn betroffen sind, ein deutlicher Effekt auf den Durchschnittslohn ausgeübt wird.

\section{Ergebnisse für die Beschäftigung}

Für die Schätzung von Beschäftigungseffekten werden zwei Maße verwendet. Auf der individuellen Ebene wird wie bei König und Möller (2008) die Weiterbeschäftigungswahrscheinlichkeit betrachtet. Da sich diese nur für Personen berechnen lässt, die vor Einführung des Mindestlohns in den Betrieben der Stichprobe beschäftigt waren, wird ergänzend die Zahl der Beschäftigten im Betrieb herangezogen. Dieses $\mathrm{Maß}$ umfasst auch Personen, die nach Einführung des Mindestlohns in die Stichprobenbetriebe eingetreten sind. ${ }^{15}$

\footnotetext{
${ }^{15}$ Prinzipiell ist es möglich, dass sich die Beschäftigtenzahl in der Branche auch infolge von Marktzutritten oder -austritten von Betrieben ändert. Auf der deskriptiven Ebene finden sich jedoch keine Hinweise für eine Veränderung der Zahl der Betriebe um den Zeitpunkt der Mindestlohneinführung (IAW 2011a, S. 211ff.), so dass diesen möglichen Beschäftigungswirkungen im Folgenden nicht nachgegangen wird.
}

Die Weiterbeschäftigungswahrscheinlichkeit im selben Betrieb $^{16}$ wird als Ergebnisvariable in einem Probit-Modell verwendet, durch das der DVD-Schätzer implementiert wird. Zur Schätzung kurz- und langfristiger Effekte werden ein- oder zweijährige Zeiträume analysiert, zu deren Beginn die Beschäftigten aus der Treatment- und Kontrollgruppe ausgewählt werden. Der Verbleib dieser Personen am Ende des Zeitraums dient dann als Ergebnisvariable. Als weiterbeschäftigt gelten hier Personen, die am Ende des Zeitraums im gleichen Betrieb beschäftigt sind wie zu Beginn. Abbildung 6 verdeutlicht für das Beispiel einer kurzfristigen Effektmessung, wie die Zeitpunkte der Zeitintervalle gewählt wurden. Der erste Zeitraum endet drei Monate vor dem Ereignis (also am 1. September 2003), was gleichzeitig den Beginn des zweiten Zeitraums darstellt, welcher damit den Zeitpunkt des natürlichen Experiments überspannt. Die langfristige Analyse erweitert die entsprechenden Zeiträume um jeweils 12 Monate früher bzw. später. Angesichts des zyklischen Geschäftsverlaufs im Maler- und Lackiererhandwerk ist entscheidend, dass die Messzeiträume die gleichen Kalendermonate umfassen.

Die Abb. 7 und 8 zeigen eine deskriptive Analyse für die kurzfristige, also im Zeitraum von einem Jahr gemessene Weiterbeschäftigungswahrscheinlichkeit. Im Zeitraum von 2001 bis $2008^{17}$ lässt sich im Maler- und Lackierer-

\footnotetext{
${ }^{16}$ Eine alternative Definition wäre die Weiterbeschäftigung innerhalb des Geltungsbereichs des Mindestlohns. Dies kann für die Kontrollgruppe jedoch nicht nachgebildet werden, da keine entsprechende Abgrenzung für Branchen außerhalb des Geltungsbereichs vorhanden ist.

${ }^{17}$ Da die erste Ziehung 2001 stattfand, können erst ab dann verlässliche Werte berechnet werden, da in den Jahren davor nur Betriebe enthalten sind, die zumindest bis 2001 überlebt haben.
} 


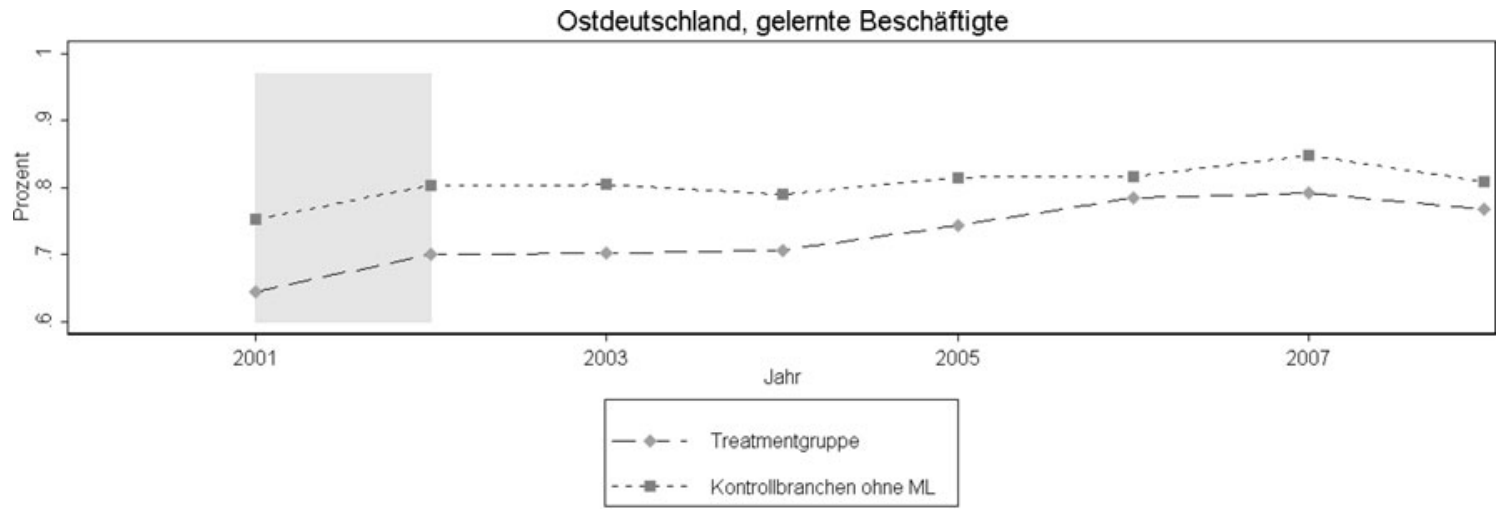

Ostdeutschland, ungelernte Beschäftigte

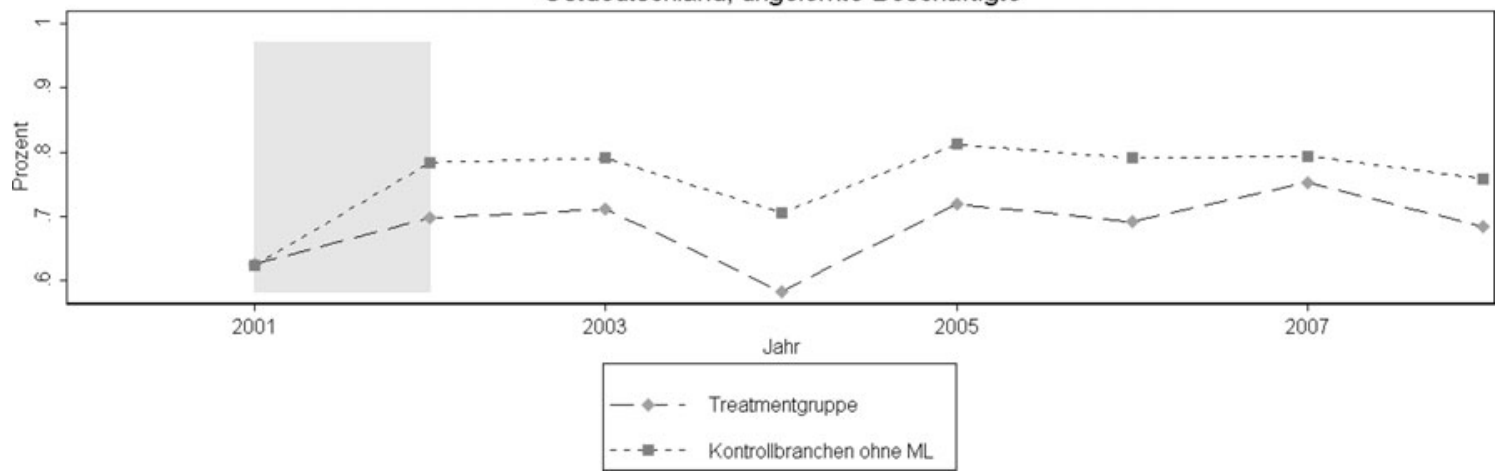

Abb. 7 Verlauf der Weiterbeschäftigungswahrscheinlichkeit, Ostdeutschland. Quelle: IEB
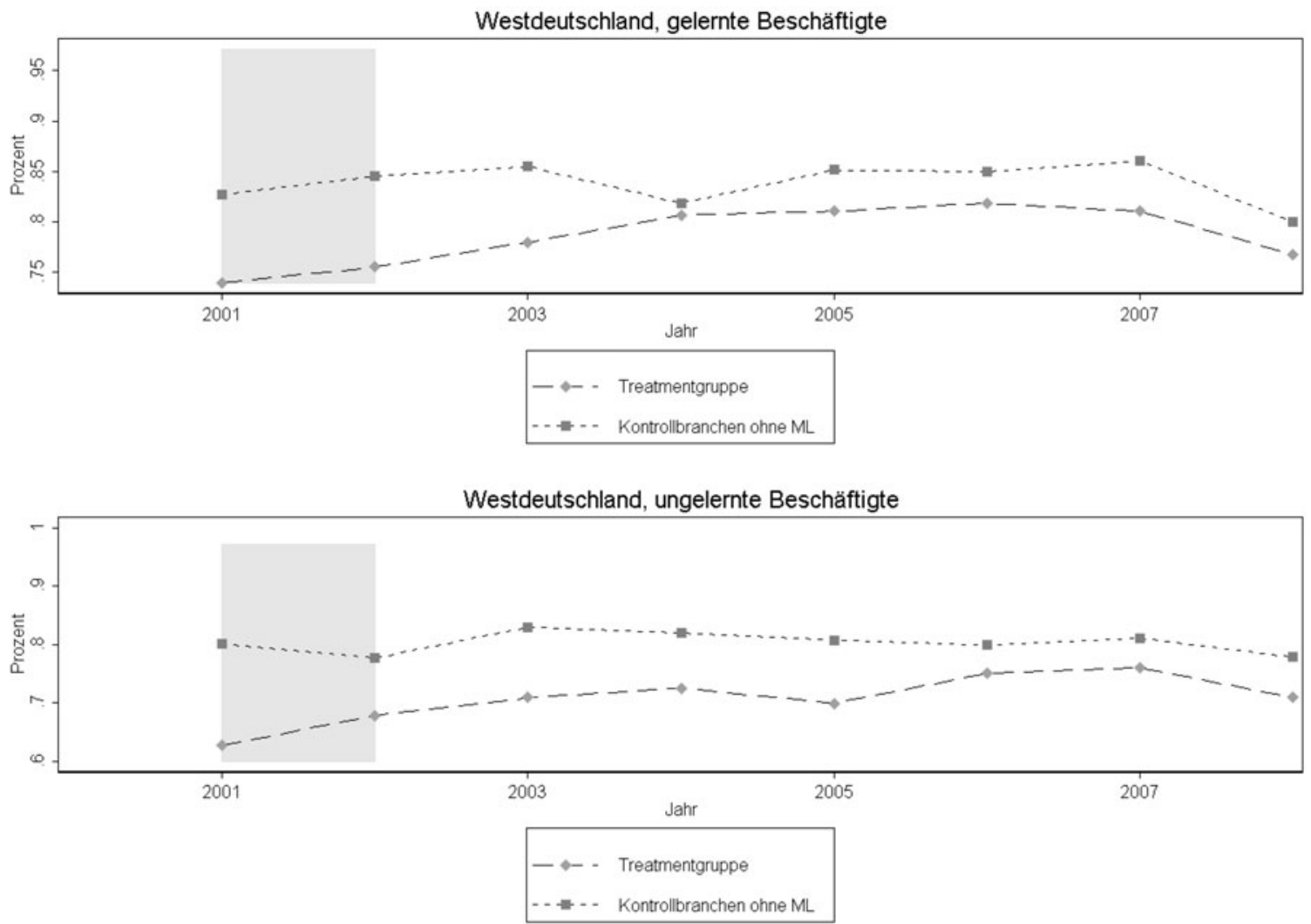

Abb. 8 Verlauf der Weiterbeschäftigungswahrscheinlichkeit, Westdeutschland. Quelle: IEB 
Tab. 3 Wirkung der Einführung des Mindestlohns auf die Weiterbeschäftigung

\begin{tabular}{|c|c|c|c|c|}
\hline & \multicolumn{2}{|l|}{ Kurze Frist } & \multicolumn{2}{|l|}{ Lange Frist } \\
\hline & Gelernte & Ungelernte & Gelernte & Ungelernte \\
\hline \multicolumn{5}{|l|}{ Ostdeutschland } \\
\hline Treatmentgruppe & $-0,083^{* * *}(4,56)$ & $-0,040(0,87)$ & $-0,001(0,43)$ & $-0,009(1,61)$ \\
\hline Zeiteffekt & $0,010(0,63)$ & $0,014(0,27)$ & $-0,007^{* * *}(2,72)$ & $-0,010^{*}(1,71)$ \\
\hline Treatment-Effekt & $-0,002(0,10)$ & $0,007(0,12)$ & $-0,002(0,54)$ & $0,002(0,26)$ \\
\hline Weitere Kovariate & $\mathrm{Ja}$ & $\mathrm{Ja}$ & $\mathrm{Ja}$ & $\mathrm{Ja}$ \\
\hline Placebo-Test, $p$-Wert & 0,90 & 0,49 & $0,04^{* *}$ & 0,63 \\
\hline Anzahl der Beobachtungen & 24.231 & 2.258 & 25.942 & 2.060 \\
\hline \multicolumn{5}{|l|}{ Westdeutschland } \\
\hline Treatmentgruppe & $-0,060^{* * *}(6,11)$ & $-0,048 *(1,79)$ & $-0,004(1,45)$ & $0,004(0,82)$ \\
\hline Zeiteffekt & $0,011(1,19)$ & $0,051^{* *}(2,19)$ & $0,008^{* * *}(3,29)$ & $-0,004(0,91)$ \\
\hline Treatment-Effekt & $0,013(0,93)$ & $-0,012(0,42)$ & $-0,002(0,77)$ & $0,001(0,17)$ \\
\hline Weitere Kovariate & $\mathrm{Ja}$ & $\mathrm{Ja}$ & $\mathrm{Ja}$ & $\mathrm{Ja}$ \\
\hline Placebo-Test, $p$-Wert & 0,87 & $0,01^{* *}$ & 0,65 & $0,02^{* *}$ \\
\hline Anzahl der Beobachtungen & 47.832 & 6.255 & 49.551 & 6.362 \\
\hline
\end{tabular}

Anmerkungen: Anmerkungen: Angegeben sind marginale Effekte. Absolute $t$-Statistiken basieren auf geclusterten Standardfehlern (auf Betriebsebene) und sind unter den Koeffizienten abgetragen. Statistische Signifikanz auf dem $1 \%$-, $5 \%$ - und $10 \%$-Niveau wird durch ***, ${ }^{* *}$ und * gekennzeichnet. Der Treatment-Effekt beruht auf dem Verfahren von Puhani (2008)

handwerk eine Zunahme der Beschäftigungsstabilität feststellen. Dies gilt sowohl für gelernte als auch ungelernte Arbeitnehmerinnen und Arbeitnehmer als auch für Ost- und Westdeutschland. Lag sie zu Beginn des Zeitraumes bei den meisten Gruppen etwas oberhalb von 0,6, stieg sie bis zum Ende des Zeitraums auf Werte zwischen 0,7 und 0,8. Nur bei den gelernten Kräften in Westdeutschland hatte die Weiterbeschäftigungswahrscheinlichkeit schon im Jahr 2001 einen entsprechend hohen Wert.

Der grau unterlegte Bereich ist für die Validität der Annahme eines gemeinsamen Trends vor dem Treatmentzeitpunkt maßgeblich. Auch hier muss bei der Beurteilung hinsichtlich eines gemeinsamen Trends zwischen gelernten und ungelernten Beschäftigten unterschieden werden. Während zwischen den beiden Zeiträumen vor Einführung des Mindestlohns für gelernte Beschäftigte die Weiterbeschäftigungswahrscheinlichkeit in beiden Gruppen im gleichen $\mathrm{Ma} ß$ angestiegen ist, unterscheidet sich der Verlauf für ungelernte Beschäftigte zwischen Treatment- und Kontrollgruppe teilweise deutlich.

Tabelle 3 enthält die durchschnittlichen marginalen Effekte auf die Weiterbeschäftigungswahrscheinlichkeit und ist gleich aufgebaut wie Tab. 2 für die Lohneffekte. Der geschätzte Treatment-Effekt und sein Standardfehler beruhen auf dem Verfahren von Puhani (2008).

Für den Treatment-Effekt werden weder in Ost- noch in Westdeutschland signifikante Auswirkungen in einer der beiden Qualifikationsgruppen festgestellt. Diesbezüglich gibt es auch keine Unterschiede zwischen der kürzer- und der längerfristigen Betrachtung. Die weiteren Ergebnisse zeigen für Ostdeutschland einen signifikant negativen Zeiteffekt für gelernte Beschäftigte in der längeren Betrachtung, in Westdeutschland dagegen einen positiven Effekt. Mit 0,7 bzw. 0,8 Prozentpunkten fallen diese Zeiteffekte aber sehr gering aus. Wie bereits aus Abb. 7 und 8 zu erkennen ist, hat die Kontrollgruppe außer bei den Ungelernten in Ostdeutschland vor dem Treatment eine höhere Weiterbeschäftigungswahrscheinlichkeit.

Die Placebo-Experimente, die für ein Pseudo-Treatment im Jahr 2001 durchgeführt wurden, deuten auf Schwachpunkte der Analyse hin. Dies trifft naturgemäß eher auf die lange Sicht als auf die kurze Sicht zu. In Ostdeutschland findet sich ein signifikanter Effekt des „Pseudo-Treatments“ für die gelernten Kräfte, in Westdeutschland für die ungelernten. In der letztgenannten Gruppe ergibt sich auch für die kurzfristige Analyse eine signifikante Placebo-Wirkung. Daher sollten die Ergebnisse mit Zurückhaltung interpretiert werden. Allerdings ergeben sich auch bei den zur Prüfung der Robustheit verwendeten anderen Kontrollgruppen keine signifikanten Treatment-Effekte (IAW 2011a, S. 200ff.).

Mögliche Wirkungen auf die Zugänge in Betriebe des Maler- und Lackiererhandwerks werden bei diesem Ansatz nicht erfasst. Beschäftigungswirkungen könnten sich aber auch dadurch eingestellt haben, dass nach der Einführung des Mindestlohns weniger Neueinstellungen vorgenommen wurden. Alternativ könnte der Mindestlohn das Arbeiten in der Branche attraktiver gemacht und über einen Angebotseffekt die Beschäftigung in der Branche erhöht haben. Beides 
Tab. 4 Wirkung der Einführung des Mindestlohns auf die Zahl der Beschäftigten im Betrieb

\begin{tabular}{llllll}
\hline & \multicolumn{2}{l}{ Ostdeutschland } & & \multicolumn{2}{c}{ Westdeutschland } \\
& Kurze Frist & Lange Frist & & Kurze Frist & Lange Frist \\
\hline Treatmentgruppe & $0,16^{* *}(2,66)$ & $0,17^{* * *}(2,93)$ & & $-0,06(1,43)$ & $-0,05(1,19)$ \\
Zeiteffekt & $-0,05(0,78)$ & $-0,17^{* * *}(2,68)$ & & $-0,01(0,22)$ & $-0,05(1,28)$ \\
Treatment-Effekt & $-0,01(0,16)$ & $0,03(0,39)$ & & $-0,05(0,84)$ & Ja \\
Weitere Kovariate & Ja & Ja & & 0,31 & $0,01(0,12)$ \\
Placebo-Test, $p$-Wert & 0,71 & 0,51 & & 0,12 & 0,12 \\
$R^{2}$ & 0,11 & 0,11 & & 5.763 & 5.752 \\
Anzahl der Beobachtungen & 2.741 & 2.709 & & Ja \\
\hline
\end{tabular}

Anmerkungen: Absolute $t$-Statistiken sind unter den Koeffizienten abgetragen. Statistische Signifikanz auf dem $1 \%$-, $5 \%$ - und $10 \%$-Niveau wird durch ${ }^{* * *},{ }^{* *}$ und ${ }^{*}$ gekennzeichnet

würde sich nicht in der Weiterbeschäftigung niederschlagen. Deshalb ist es notwendig, ergänzend zur Weiterbeschäftigung auch den Bestand der Beschäftigung auf Betriebsebene zu analysieren. Dieser ist hier als die Anzahl Beschäftigter eines Betriebs zu einem Stichtag definiert. In einem linearen Regressionsmodell wird der Logarithmus der Beschäftigtenzahl als abhängige Variable verwendet, um unterschiedliche Betriebsgrößen angemessen zu berücksichtigen. Die Messzeitpunkte sind der 1. September 2002 und alternativ der 1. September 2004 oder 2005.

Signifikante Treatment-Effekte des Mindestlohns auf die Betriebsgröße werden in den Schätzungen nicht gefunden (siehe Tab. 4). Das bedeutet, dass der Mindestlohn nicht nur keine Auswirkungen auf die Stabilität der bestehenden Arbeitsverhältnisse gehabt zu haben scheint, sondern auch den Stand der Beschäftigung nicht beeinflusst hat. Für den durchschnittlichen Bestand an Beschäftigten ergibt sich in Ostdeutschland ein negativer Zeittrend (Tab. 4). In Ostdeutschland bedeutet die Zugehörigkeit zu der Treatmentgruppe zudem eine höhere durchschnittliche Zahl an Beschäftigten pro Betrieb. Die PlaceboExperimente für das Jahr 2001 zeigen keine Verletzung der Annahme gemeinsamer Trends zwischen Treatment- und Kontrollgruppe an.

Zusätzlich wurden die Schätzungen, die Tab. 4 zugrunde liegen, auch allein mit der Zahl der ungelernten Arbeitskräfte vorgenommen. Da es eine relative große Zahl von Betrieben gibt, die keine ungelernten Kräfte beschäftigten, wurden Tobit-Modelle geschätzt, um Randlösungen in den Schätzungen berücksichtigen zu können. Die Ergebnisse unterscheiden sich qualitativ nicht von den Ergebnissen für die Gesamtzahl der Beschäftigten und werden hier nicht präsentiert.

Schließlich wurde auch in diesem Fall eine Robustheitsanalyse mit einer Kontrollgruppe von Beschäftigten aus Mindestlohnbranchen durchgeführt. Auch hierbei fanden sich keine signifikanten Effekte.

\section{Ergebnisse aus aktuellen Befragungsdaten}

Für die deutliche Erhöhung der Mindestlöhne für die ungelernten Kräfte im Jahr 2009 konnte im Rahmen der Evaluation nur eine Analyse auf der Basis vorläufiger Daten vorgenommen werden. Diese enthalten keine Löhne, und für den Arbeitsmarktzustand ist nur eine vorläufige Messung verfügbar. Die Ergebnisse mit dem erweiterten Verbleibsnachweis zeigen keine signifikanten Beschäftigungseffekte. Dies kann aber auch an Messungenauigkeiten liegen. Diese Ergebnisse der Evaluation werden hier nicht wiedergegeben. ${ }^{18}$

Um aktuellere Ergebnisse zur Wirkung von Mindestlöhnen zu erhalten, werden stattdessen die Ergebnisse einer Betriebsbefragung herangezogen. Ziel der standardisierten Befragung war die Erhebung ergänzender repräsentativer Informationen zur Anwendung und zu den Wirkungen des Mindestlohns im Maler- und Lackiererhandwerk. Durch den Fragebogen wurden Informationen zu betrieblichen Merkmalen wie Beschäftigung, Umsatzstrukturen oder Tätigkeitsschwerpunkte erhoben. Weitere Fragen betrafen die Wettbewerbsbedingungen, die Tarifbindung und die Einschätzung der Mindestlöhne. Die Befragung wurde im Frühjahr 2011 durch das IAW in einer Bruttostichprobe von 4.000 Betrieben des Maler- und Lackiererhandwerks im schriftlichen Befragungsmodus durchgeführt und ergab einen Rücklauf von 890 beantworteten Fragebogen. Nach Einschränkung auf den betrieblichen Geltungsbereich und Qualitätskontrollen bei den Rückläufen verblieben 799 in die Auswertung einbezogene Beobachtungen. Die Stichprobe wurde disproportional nach Größenklasse gezogen. Die Ergebnisse wurden mit entsprechenden Gewichtungsfaktoren und Ausfallgewichten auf die Grundgesamtheit hochgerechnet (IAW 2011a, S. 38ff.). Die folgenden Ergebnisse der Befragung dürfen nicht als Evidenz für kausale

\footnotetext{
${ }^{18}$ Die Ergebnisse sind in IAW (2011a, S. 207ff.) aufgeführt.
} 
Abb. 9 Ungelernte

Mitarbeiterinnen und

Mitarbeiter, die mehr als den

Mindestlohn erhalten, Ost- und

Westdeutschland. Quelle:

IAW-Befragung zum

Mindestlohn im Maler- und

Lackiererhandwerk,

hochgerechnete Angaben
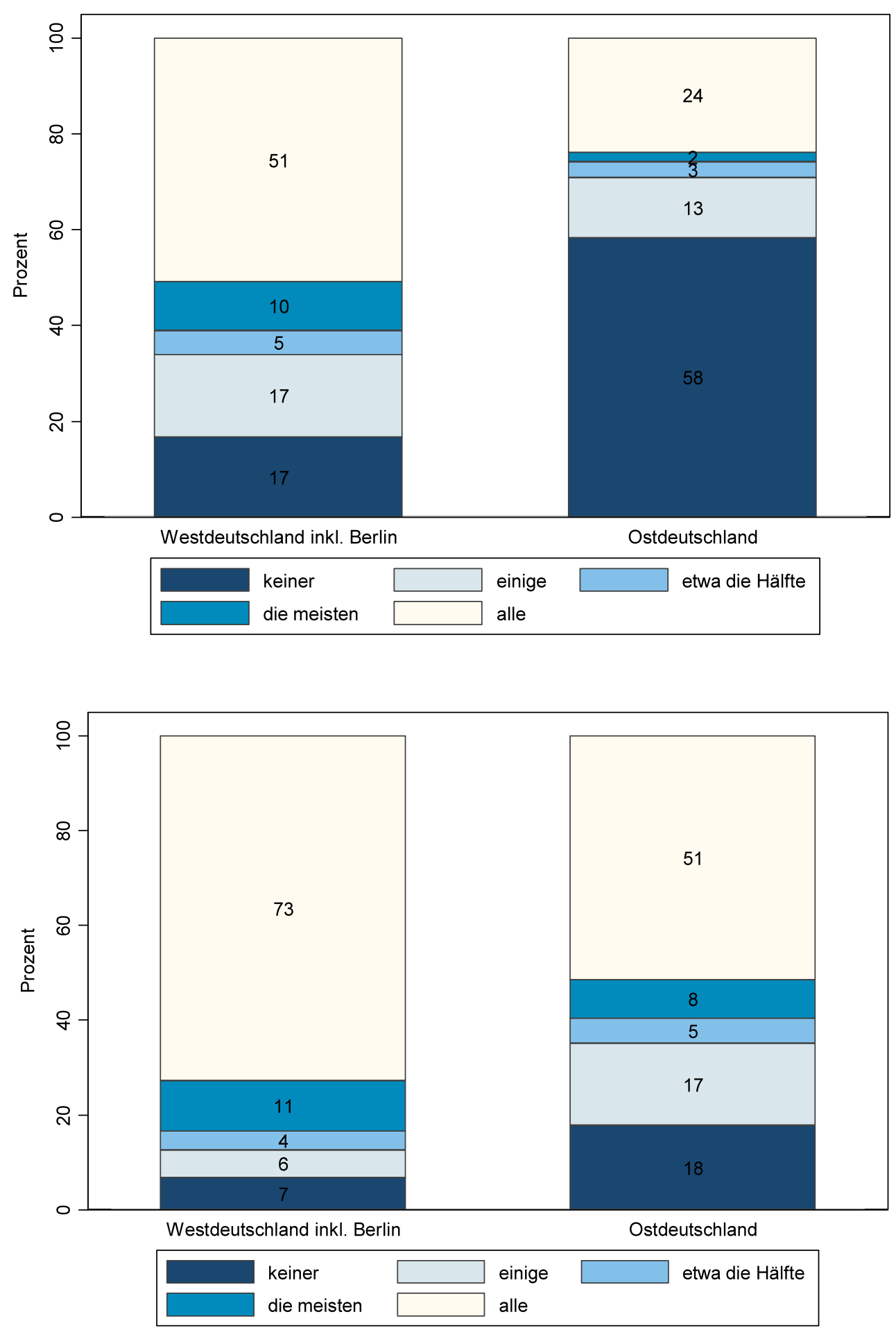

Abb. 10 Gelernte

Mitarbeiterinnen und

Mitarbeiter, die mehr als den

Mindestlohn erhalten, Ost- und

Westdeutschland. Quelle:

IAW-Befragung zum

Mindestlohn im Maler- und

Lackiererhandwerk,

hochgerechnete Angaben
Beziehungen verstanden werden, sondern stellen lediglich einen Interpretationshintergrund für die Ergebnisse der kausalen Wirkungsanalyse dar.

Die Angaben beziehen sich, wenn nicht anders gesagt, auf das Jahr 2010. Die Abb. 9 und 10 zeigen, dass die Eingriffsintensität der Mindestlöhne bei den ungelernten Kräften in diesem Jahr wesentlich höher erscheint als bei den gelernten. In dieser Hinsicht haben sich die Verhältnisse in der Branche seit dem in der Wirkungsanalyse betrachteten Zeitraum von 2003 bis 2006 deutlich geändert. Dies gilt insbesondere für Ostdeutschland, wo $58 \%$ Prozent der Betriebe keinem der ungelernten Beschäftigten mehr als den Mindestlohn bezahlen. Bei den gelernten Kräften (Abb. 10) beträgt dieser Anteil dagegen nur $18 \%$. Gut die Hälfte (51\%) 
Abb. 11 Rückgang der Beschäftigtenzahl infolge des Mindestlohns aus Sicht der Betriebe. Quelle: IAW-Befragung zum Mindestlohn im Maler- und Lackiererhandwerk, hochgerechnete Angaben

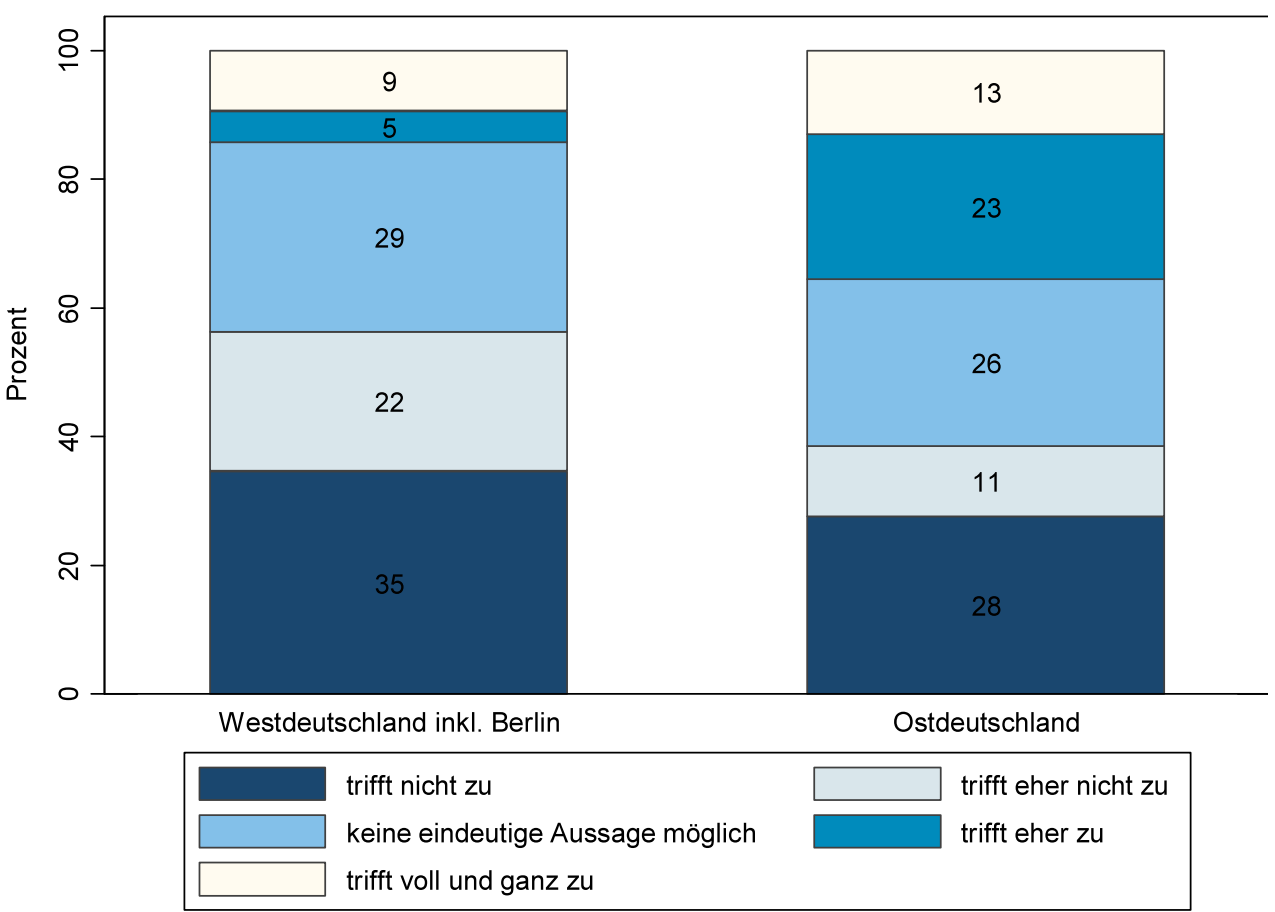

der ostdeutschen Betriebe bezahlen allen gelernten Kräften mehr als den Mindestlohn. Insofern gibt es keine Anzeichen für eine Kompression der Löhne auf dem Niveau des Mindestlohns. In Westdeutschland ist der Prozentsatz der Betriebe, die ausschließlich oberhalb des Mindestlohns entlohnen, jeweils deutlich höher als in Ostdeutschland; das Gegenteil gilt für Betriebe, die alle Beschäftigten lediglich nach dem Mindestlohn bezahlen.

Die wahrgenommenen Wirkungen auf die Beschäftigung zeigt Abb. 11. Der Aussage, dass der Mindestlohn im Malerund Lackiererhandwerk insgesamt (also nicht nur im befragten Betrieb und nicht nur im Jahr 2010) zu einem Rückgang der Zahl der Beschäftigten geführt hat, stimmen in Ostdeutschland mit $36 \%$ erheblich mehr Betriebe zu als in Westdeutschland (14\%), während $39 \%$ bzw. $57 \%$ diese Aussage ablehnen. Zugleich ist festzuhalten, dass auch in Ostdeutschland und selbst nach der deutlichen Erhöhung im Jahr 2009 nur ein gutes Drittel der Betriebe negative Beschäftigungswirkungen der Mindestlöhne wahrnimmt. ${ }^{19}$

Lassen sich den Befragungsergebnissen systematische Gründe entnehmen, weshalb sich in den statistischen Analysen keine Beschäftigungseffekte finden und weshalb diese auch von den Betroffenen mehrheitlich nicht erwartet werden, obwohl dies nach der neoklassischen Sichtweise des Arbeitsmarktes zu erwarten wäre? Im Folgenden sollen drei

\footnotetext{
${ }^{19}$ Die Frage im Fragebogen bezieht sich dabei auf die Wirkungen insgesamt, ohne zwischen der ferneren und der näheren Vergangenheit zu unterscheiden.
}

mögliche Gründe mit Hilfe der Befragungsdaten auf ihre Relevanz geprüft werden: die mögliche Kompensation der Mindestlöhne durch Ausweichreaktionen, die Möglichkeit der Überwälzung gestiegener Personalkosten und das Monopsonargument.

Beschäftigungswirkungen könnten sich deshalb nicht einstellen, weil der Anstieg der Personalkosten durch die Mindestlöhne dadurch begrenzt wird, dass als Kompensation freiwillige Leistungen des Arbeitgebers gekürzt werden oder ganz wegfallen. Möglich ist dies, wenn diese Leistungen nicht regelmäßig gewährt werden und damit dem für die Anwendung des Mindestlohns relevanten Entgelt nicht zugerechnet werden. Ungefähr ein Drittel der ostdeutschen Betriebe ist der Meinung, dass eine solche Kompensation im Maler- und Lackiererhandwerk stattgefunden hat (Abb. 12). Ganz offensichtlich reicht diese Kompensation aber nicht aus, um die Wirkungen des Mindestlohns auf die Personalkosten zu neutralisieren, denn in Ostdeutschland sind $63 \%$ der Betriebe der Auffassung, dass der Mindestlohn zu einer Erhöhung der Personalkosten im Maler- und Lackiererhandwerk geführt hat (Abb. 13). In Westdeutschland stimmen dagegen nur ein Viertel der Betriebe dieser Aussage zu, was mit der wesentlich geringeren Eingriffsintensität des Mindestlohns in den alten Bundesländern übereinstimmt.

Der Grad, in dem gestiegene Personalkosten an die Kunden weitergegeben werden können, ist eine wesentliche Determinante der Lohnkostenelastizität der Beschäftigung. Exakte Information auf der betrieblichen Ebene über die Preise der vom Maler- und Lackiererhandwerk erbrachten Dienst- 
Abb. 12 Wegfallen freiwilliger Leistungen infolge des Mindestlohns. Quelle: IAW-Befragung zum Mindestlohn im Maler- und Lackiererhandwerk, hochgerechnete Angaben

Abb. 13 Erhöhung der Personalkosten durch den Mindestlohn nach Einschätzung der Betriebe. Quelle: IAW-Befragung zum Mindestlohn im Maler- und Lackiererhandwerk, hochgerechnete Angaben
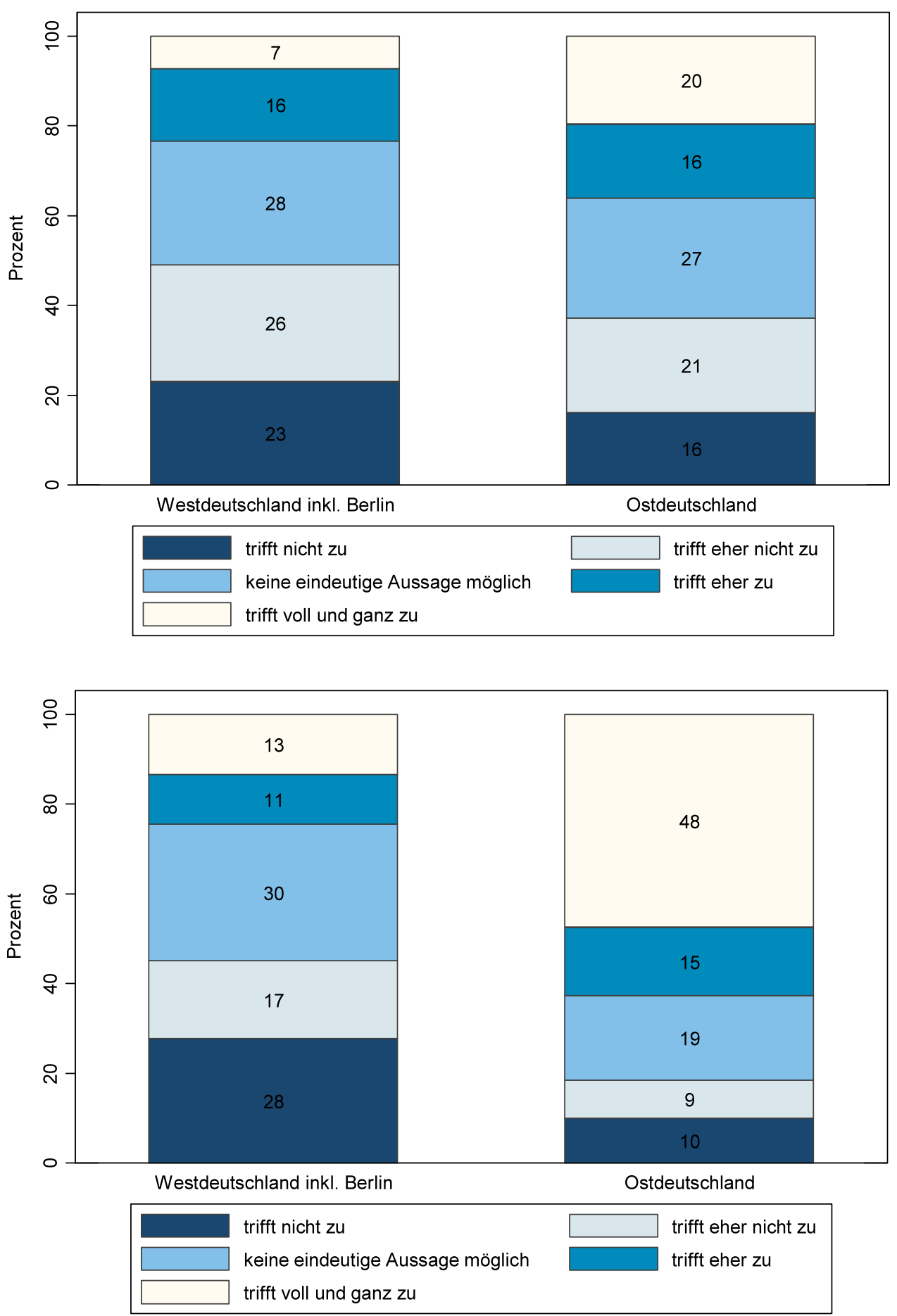

leistungen steht nicht zur Verfügung; es kann lediglich indirekte Evidenz zur Wettbewerbssituation auf dem Absatzmarkt benutzt werden. ${ }^{20}$ Bei differenzierter Betrachtung

\footnotetext{
${ }^{20}$ In den qualitativen Befragungen, die im Rahmen des Projekts durchgeführt wurden, wurde die Überwälzung in höhere Preise allerdings wiederholt als reale Möglichkeit geäußert (siehe IAW 2011a, S. 222ff.).
}

zwischen Ost- und Westdeutschland zeigt sich, dass die in den neuen Bundesländern angesiedelten Maler- und Lackiererbetriebe einen höheren Wettbewerbsdruck wahrnehmen als die Betriebe in den alten Bundesländern (siehe Abb. 14). Vor allem die Kategorie ,sehr hoher Wettbewerbsdruck“ ist im Osten mit $45 \%$ deutlich häufiger vertreten als im Westen $(32 \%)$. Insgesamt beschreiben sechs von zehn Betrieben in Ostdeutschland den Wettbewerbsdruck mindestens als er- 
Abb. 14 Wahrnehmung der Wettbewerbssituation aus Sicht der Betriebe. Quelle: IAW-Befragung zum Mindestlohn im Maler- und Lackiererhandwerk, hochgerechnete Angaben

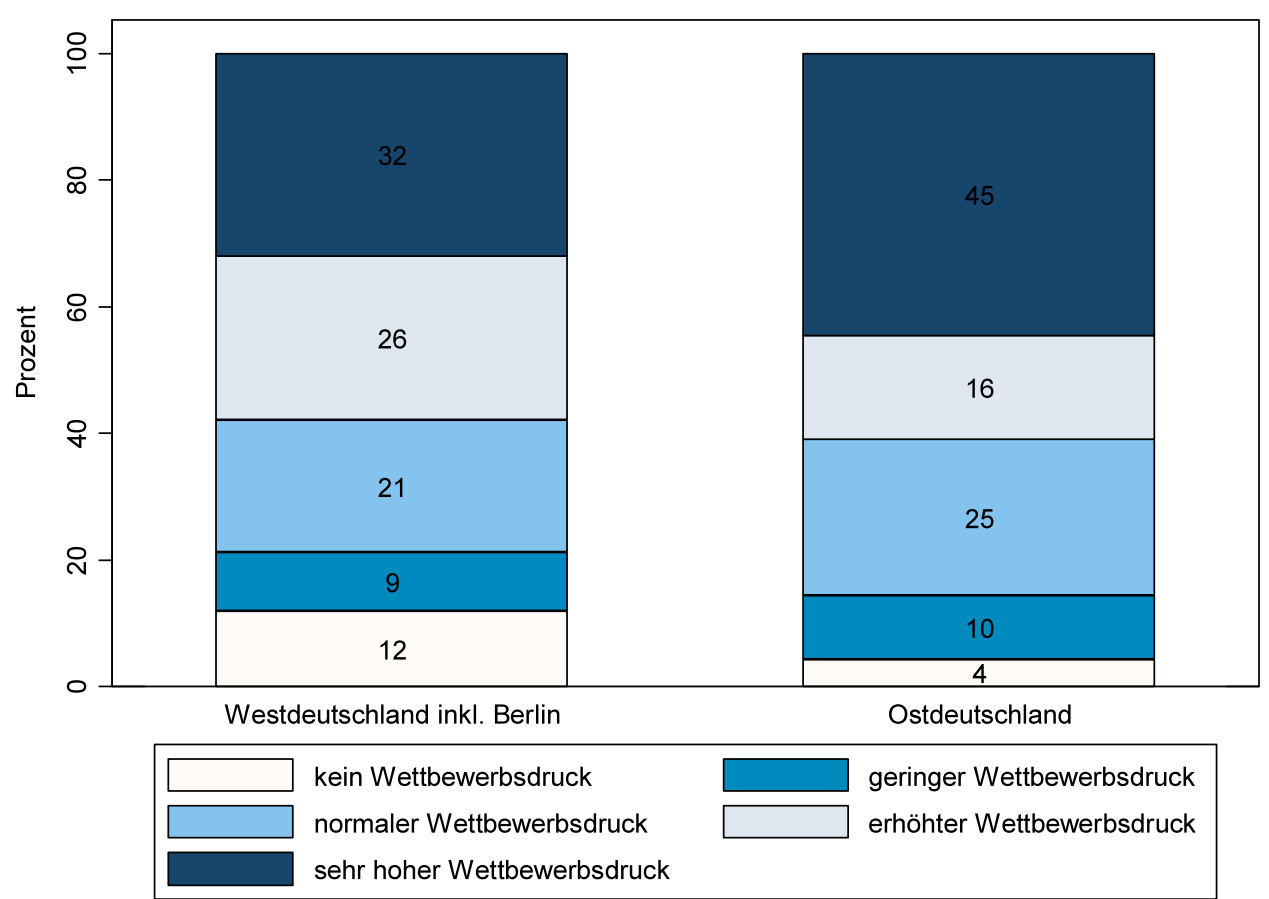

höht. Insbesondere wenn der Wettbewerb durch Anbieter entsteht, die nicht dem Mindestlohn unterliegen (sogenannte „Alleinmeister“, do-it-yourself-Tätigkeiten und Schattenwirtschaft), begrenzt dies den Spielraum zur Überwälzung der gestiegenen Kosten auf die Nachfrageseite.

Ein unvollkommener Wettbewerb kann auch auf dem Arbeitsmarkt bestehen. Der Fall des Monopsons ist in der Literatur der Paradefall für eine Wettbewerbsverzerrung, die durch einen in der Höhe richtig gesetzten Mindestlohn korrigiert werden kann (siehe z.B. Flinn 2010). Im Monopsonfall haben die Betriebe Lohnsetzungsspielräume, weil es für die Arbeitsanbieter nicht oder nur eingeschränkt die Möglichkeit gibt, zu anderen Betrieben zu wechseln. Wenn ein Betrieb seine Arbeitsnachfrage senkt, wird durch den vermehrten Wettbewerb unter den Arbeitsanbietern ein negativer Anpassungsdruck auf die Löhne erzeugt. Anders ausgedrückt: die Arbeitsangebotsfunktion für den einzelnen Betrieb ist aufwärts geneigt. Ein Mindestlohn beseitigt den Anreiz zur Senkung der Arbeitsnachfrage und erhöht damit tendenziell die Beschäftigung.

In der Befragung wurde direkt erhoben, ob die Arbeitsangebotsfunktion aufwärts geneigt ist. Die entsprechende Frage lautete, ob der Mindestlohn ausreicht, um geeignete neue Arbeitskräfte zu gewinnen. In diesem Fall ist die Arbeitsangebotsfunktion für den Betrieb horizontal, monopsonistische Marktmacht besteht also nicht. Im Fragebogen wurde dabei zwischen gelernten und ungelernten Arbeitskräften unterschieden. Hinsichtlich der ersten Gruppe ist gut die Hälfte der ostdeutschen Betriebe der Meinung, dass der Mindestlohn ausreicht, um weitere Arbeitskräfte zu gewinnen; in Westdeutschland sind dagegen nur $18 \%$ der Betriebe dieser Auffassung. Für die ungelernten Kräfte gilt in Ostund Westdeutschland, dass die Höhe des Mindestlohns aus Sicht der weitaus meisten Betriebe zur Rekrutierung neuer Arbeitskräfte ausreichend ist.

Beim geltenden Mindestlohn ist also zumindest bei den ungelernten Kräften gesichert, dass sich die Betriebe nicht wie Monopsonnachfrager verhalten können. Wenn die Betriebe - was nicht geprüft werden kann - in diesem Segment in der hypothetischen Situation ohne den Mindestlohn Monopsonmacht auf dem Arbeitsmarkt hätten, wäre daraus zu erklären, dass der Mindestlohn keine negativen Beschäftigungswirkungen hat.

Bei den gelernten Arbeitskräften ist es zu vermuten, dass der Wettbewerb um Arbeitskräfte die Marktmacht der Nachfrager auf dem Arbeitsmarkt begrenzt. Nach den Ergebnissen der Befragung hat sich die Konkurrenz der Betriebe um Fachkräfte in den letzten fünf Jahren verschärft. Erschwerte Rekrutierungsbedingungen ergeben sich dabei vor allem in Ostdeutschland, wo $66 \%$ der Betriebe diese Bedingungen feststellen. Monopsonistische Marktstrukuren scheinen deshalb in diesem Bereich des Arbeitsmarktes derzeit unwahrscheinlich, wenngleich diese für regionale oder fachliche Teilmärkte nicht auszuschließen sind. Erklärungsbedürftig bleibt allerdings, weshalb die Konkurrenz um Fachkräfte offenbar keinen starken Aufwärtsdruck auf die Löhne ausübt; vielmehr konzentriert sich bei den gelernten Kräften die Lohnverteilung stark beim Niveau des Mindestlohns (siehe Abb. 4).

In der neueren Literatur gibt es neben dem statischen Lehrbuchmodell des Monopsons noch weitere Gründe für 
unvollkommenen Wettbewerb auf dem Arbeitsmarkt, bei deren Geltung ein bindender Mindestlohn ebenfalls keine negativen Beschäftigungswirkungen hat. Insbesondere könnten Suchfriktionen ökonomische Renten erzeugen, über deren Verteilung Betrieb und Beschäftigte verhandeln, wenn sie die Löhne festsetzen (Flinn 2010: 43ff.; Manning 2003). Neben den anderen diskutierten Gründen könnte das Monopsonargument Teil einer Erklärung sein, weshalb bislang keine Evidenz für deutliche Beschäftigungsverluste durch den Mindestlohn besteht.

\section{Schlussfolgerungen}

Ziel der Evaluation war es nach dem Koalitionsvertrag der Regierungsparteien im Bund aus dem Jahr 2009, die bestehenden gesetzlichen Mindestlöhne dahingehend zu überprüfen, ob sie Arbeitsplätze gefährden oder neuen Beschäftigungsverhältnissen entgegenstehen und ob sie sowohl den erforderlichen Schutz der Arbeitnehmer als auch die Wettbewerbsfähigkeit der einzelnen Branchen gewährleisten. Die Evaluation strebte daher ein Höchstmaß an interner Validität für die betrachteten Branchen an, während die externe Validität hinsichtlich anderer Branchen keine Rolle spielte. Daher beschränken sich die Schlussfolgerungen auf das Maler- und Lackiererhandwerk.

Die quantitative Evidenz zur Wirkung der Mindestlöhne im Maler- und Lackiererhandwerk auf die Lohnhöhe basiert auf dem natürlichen Experiment, das durch die Einführung des Mindestlohns im Jahr 2003 hervorgerufen wurde. Hierbei gibt es deutliche Unterschiede zwischen Ost- und Westdeutschland. In Westdeutschland hatte der Mindestlohn bei der Einführung eine sehr geringe Eingriffsintensität. In Ostdeutschland ist die Eingriffsintensität der Mindestlöhne dagegen weitaus höher. Hierbei muss man allerdings zwischen gelernten und ungelernten Kräften unterscheiden. Besonders hoch im Vergleich zu den vor Einführung des Mindestlohns gezahlten effektiven Löhnen ist der Mindestlohn für gelernte Kräfte. Etwa die Hälfte der Beschäftigten in dieser Kategorie war 2003 direkt vom Mindestlohn betroffen. Im Bereich der ungelernten Kräfte galt dies für einen wesentlich geringeren Anteil der Arbeitskräfte. Entsprechend diesen deskriptiven Befunden lassen sich positive und statistisch signifikante Treatment-Effekte auf den Durchschnittslohn nur für gelernte Kräfte in Ostdeutschland feststellen.

Begrenzt wird die Lohnwirkung durch eine partielle Nichteinhaltung der Mindestlohnbestimmungen. Nach den statistischen Ergebnissen erhielten $15 \%$ der gelernten Arbeitskräfte in Ostdeutschland im Jahr 2006 weniger als den für sie geltenden Mindestlohn. Auch die qualitativen Befragungen, die im Rahmen des Projekts durchgeführt wurden, zeigen, dass die Anwendung der Mindestlöhne in den ostdeutschen Betrieben teilweise unvollkommen war. Die Schwierigkeiten bei der Abgrenzung von gelernten und ungelernten Arbeitskräften wurden daher auch als Grund dafür genannt, dass der Mindestlohn 2009 im Osten auf einheitlich 9,50 Euro angehoben wurde (IAW 2011a, S. 225).

Im Bereich der ungelernten Arbeitskräfte hat sich die Eingriffsintensität nach der Erhöhung der Mindestlöhne im Jahr 2009 deutlich erhöht. Die Ergebnisse der standardisierten Befragung zeigen, dass nunmehr ein hoher Anteil der ungelernten Kräfte direkt vom Mindestlohn betroffen ist. $\mathrm{Da}$ auch die gelernten Arbeitskräfte in Ostdeutschland oft nur den Mindestlohn bekommen, wird das Lohndifferential zwischen gelernten und ungelernten Kräften eingeebnet und eine differenzierte Entlohnung von gelernten und ungelernten Arbeitnehmern erschwert. Dies wirkt längerfristig darauf hin, dass die Anreize, eine Berufsausbildung zu durchlaufen, abnehmen.

Die Beschäftigungseffekte des Mindestlohns werden anhand der Einführung im Jahr 2003 untersucht. Weder für gelernte noch für ungelernte Arbeitskräfte wird Evidenz für Beschäftigungswirkungen gefunden; dies gilt gleichermaBen für Ost- und Westdeutschland und für unterschiedliche Arten, die Beschäftigung zu messen. Hierbei ist zu berücksichtigen dass die Entsendung von Arbeitnehmerinnen und Arbeitnehmern aus dem Ausland nicht berücksichtigt werden konnte, da hierfür derzeit keine Daten zur Verfügung stehen Die qualitative Evidenz lässt darauf schließen, dass der Mindestlohn die Arbeitnehmerentsendung begrenzt hat. Danach gab es insbesondere im Bereich der Objektbetreuung und bei größeren Unternehmen einen starken Wettbewerb durch ausländische Anbieter. Mit der Einführung des Mindestlohns sind diese Substitutionsmöglichkeiten begrenzt worden, was die Wettbewerbsfähigkeiten der inländischen Anbieter erhöhte (IAW 2011a: 252).

Auch die deutliche Erhöhung der Mindestlöhne für ungelernte Beschäftigte im Jahr 2009 könnte als natürliches Experiment zur Feststellung von Beschäftigungswirkungen verwendet werden. Zum Zeitpunkt der Evaluation lag jedoch noch keine ausreichende Datenbasis für den Zeitraum nach der Erhöhung vor, um belastbare Ergebnisse zu möglichen Wirkungen zu erhalten. Insgesamt ist in Ostdeutschland ein Drittel der Betriebe der Auffassung, dass der Mindestlohn negative Beschäftigungswirkungen hat. Auch in den qualitativen Interviews wurde seitens unterschiedlicher Akteure geäußert, dass die Beschäftigungssituation ungelernter Arbeitskräfte nach 2009 in Ostdeutschland schwieriger geworden ist (IAW 2011a, S. 225f.). Daher ist weitere Forschung angezeigt, sobald die entsprechenden Daten zur Verfügung stehen. Da es teilweise Anzeichen dafür gibt, dass bei den ungelernten Kräften die Annahme der gemeinsamen Trends zwischen der hier verwendeten Kontrollgruppe und der Treatmentgruppe nicht erfüllt sein könnte, sollte hierbei auch die Wahl der Kontrollgruppen noch einmal thematisiert werden. 


\section{Executive summary}

Minimum wages were first introduced in the German painting and varnishing trades in December 2003. They are differentiated according to region (East and West Germany) and skill level (skilled and unskilled workers); however, the skill differential was abolished in 2009 in East Germany. In this paper, we present key findings from the final report on the evaluation of the minimum wage in the painting and varnishing trades, which was conducted on behalf of the German Federal Government.

For the analysis, the difference-in-differences method is used. It is based on the natural experiment that was caused by the introduction of the minimum wage in 2003 . The control group was drawn from those sectors of the construction industry that did not have a minimum wage over the entire study period.

In West Germany, the minimum wage has a low level of intervention. In East Germany, the intensity of the minimum wage, however, is much stronger. Compared to the wages paid prior to the introduction of the minimum wage, the minimum wage for skilled workers is particularly high in this part of the country. About half of the employees in this category were directly affected by the minimum wage in 2003 in the sense that their previous wage was lower than the minimum wage. Amongst unskilled workers, this was true for a much smaller proportion of the workforce. Consistent with these descriptive findings, we find positive and statistically significant treatment effects on the average wage for skilled workers in East Germany. The wage effect of the minimum wage is reduced by partial non-compliance. According to our statistical results, $15 \%$ of skilled workers in East Germany in 2006 were remunerated at a rate less than the applicable minimum wage.

Regarding the employment effects of the minimum wage, we find no statistically significant evidence, neither for skilled nor for unskilled workers. This finding applies equally to East and West Germany. Employment is measured as the probability of continued employment in the company. Alternatively, the stock of employment at the company level is analyzed. We find no statistically significant treatment effects for either of these variables.

\section{Kurzfassung}

Im deutschen Maler- und Lackiererhandwerk wurden erstmals zum 1. Dezember 2003 Mindestlöhne nach dem Arbeitnehmer-Entsendegesetz eingeführt. Die Mindestlöhne im Maler- und Lackiererhandwerk sind nach Ost- und Westdeutschland differenziert und haben derzeit in Westdeutschland zwei Stufen für gelernte und ungelernte Arbeitskräfte, während diese Unterscheidung seit 2009 in Ost- deutschland entfallen ist. In diesem Beitrag werden wichtige Ergebnisse aus dem Abschlussbericht des Instituts für Angewandte Wirtschaftsforschung (IAW) zur Evaluation der Mindestlöhne im Maler- und Lackiererhandwerk wiedergegeben, die im Auftrag der Bundesregierung durchgeführt wurde.

Für die Wirkungsanalyse wird das Differenz-von-Differenzen-Verfahren verwendet. Es basiert auf dem natürlichen Experiment, das durch die Einführung des Mindestlohns im Jahr 2003 hervorgerufen wurde. Die Kontrollgruppe wurde aus Branchen des Baugewerbes gezogen, für die über den gesamten Untersuchungszeitraum kein Mindestlohn galt.

In Westdeutschland hat der Mindestlohn eine geringe Eingriffsintensität. In Ostdeutschland ist die Eingriffsintensität der Mindestlöhne dagegen weitaus höher. Hierbei muss man allerdings zwischen gelernten und ungelernten Kräften unterscheiden. Besonders hoch im Vergleich zu den vor Einführung des Mindestlohns gezahlten effektiven Löhnen ist der Mindestlohn für gelernte Kräfte. Etwa die Hälfte der Beschäftigten in dieser Kategorie war 2003 direkt vom Mindestlohn betroffen. Im Bereich der ungelernten Kräfte galt dies für einen wesentlich geringeren Anteil der Arbeitskräfte. Entsprechend diesen deskriptiven Befunden lassen sich positive und statistisch signifikante TreatmentEffekte auf den Durchschnittslohn nur für gelernte Kräfte in Ostdeutschland feststellen. Begrenzt wird die Lohnwirkung durch eine partielle Nichteinhaltung der Mindestlohnbestimmungen. Nach den statistischen Ergebnissen erhielten $15 \%$ der gelernten Arbeitskräfte in Ostdeutschland im Jahr 2006 weniger als den für sie geltenden Mindestlohn.

Bei den Beschäftigungseffekten des Mindestlohns wird weder für gelernte noch für ungelernte Arbeitskräfte Evidenz für Beschäftigungswirkungen gefunden; dies gilt gleichermaßen für Ost- und Westdeutschland. Die Beschäftigung wird zum einen als Wahrscheinlichkeit der Weiterbeschäftigung im Betrieb gemessen. Zum anderen wird der Bestand der Beschäftigung auf Betriebsebene analysiert. Für keine dieser Größen finden sich statistisch signifikante Treatment-Effekte.

Danksagung Dieser Beitrag entstand im Rahmen der Evaluation bestehender gesetzlicher Mindestlohnregelungen, welche vom Ministerium für Arbeit und Soziales (BMAS) in Auftrag gegeben wurde. Die Ergebnisse geben nicht unbedingt die Meinung des BMAS wieder. Wir danken für die hilfreichen Kommentare eines anonymen Gutachters oder einer Gutachterin sowie des Herausgebers. Wir bedanken uns ferner beim Institut für Arbeitsmarkt- und Berufsforschung (IAB), Nürnberg und dem Statistischen Landesamt Baden-Württemberg für die Bereitstellung der Daten. Katrin Harsch, Günther Klee, Andreas Koch, Raimund Krumm, Jochen Späth, Harald Strotmann und Hans Verbeek haben wertvolle Beiträge zu dieser Arbeit geleistet. Darüber hinaus wurden wir von einem engagierten Team studentischer Hilfskräfte unterstützt. Ihnen allen sei herzlich gedankt; verbleibende Fehler liegen ausschließlich in der Verantwortung der Autoren. 


\section{Anhang: Tabelle 5}

Tab. 5 Mittelwerte der unabhängigen Variablen

\begin{tabular}{|c|c|c|c|}
\hline & Kontrollgruppe & Treatmentgruppe & $p$-Wert \\
\hline \multicolumn{4}{|l|}{ Persönliche Merkmale } \\
\hline Männlich & 0,966 & 0,979 & 0,000 \\
\hline Keine Berufsausbildung & 0,044 & 0,042 & 0,257 \\
\hline Berufsausbildung & 0,933 & 0,948 & 0,000 \\
\hline Abitur & 0,004 & 0,002 & 0,015 \\
\hline Abitur und Berufsausbildung & 0,019 & 0,008 & 0,000 \\
\hline Unter 30 Jahre & 0,225 & 0,261 & 0,000 \\
\hline 30-39 Jahre & 0,318 & 0,302 & 0,001 \\
\hline 40-49 Jahre & 0,282 & 0,266 & 0,000 \\
\hline 50-59 Jahre & 0,139 & 0,149 & 0,007 \\
\hline Über 60 Jahre & 0,035 & 0,022 & 0,000 \\
\hline Staatsangehörigkeit: Deutschland & 0,962 & 0,943 & 0,000 \\
\hline Staatsangehörigkeit: EU (exkl. Osteuropa), andere Industrieländer & 0,013 & 0,019 & 0,000 \\
\hline Staatsangehörigkeit: Osteuropa (exkl. früheres Jugoslawien) & 0,004 & 0,005 & 0,150 \\
\hline Staatsangehörigkeit: sonstige Länder & 0,021 & 0,033 & 0,000 \\
\hline Betriebszugehörigkeit 1. Quartil & 0,176 & 0,293 & 0,000 \\
\hline Betriebszugehörigkeit 2. Quartil & 0,257 & 0,243 & 0,002 \\
\hline Betriebszugehörigkeit 3. Quartil & 0,282 & 0,234 & 0,000 \\
\hline Betriebszugehörigkeit 4. Quartil & 0,285 & 0,230 & 0,000 \\
\hline Auszubildender & 0,002 & 0,002 & 0,314 \\
\hline Vollzeit & 0,957 & 0,994 & 0,000 \\
\hline Große Teilzeit ( $\geq 15$ Stunden/Woche) & 0,004 & 0,002 & 0,000 \\
\hline Kleine Teilzeit (<15 Stunden/Woche) & 0,001 & 0,001 & 0,613 \\
\hline Geringfügig beschäftigt & 0,036 & 0,000 & 0,000 \\
\hline Nebentätigkeit & 0,006 & 0,001 & 0,000 \\
\hline \multicolumn{4}{|l|}{ Regionalmerkmale } \\
\hline Landkreis & 0,641 & 0,610 & 0,000 \\
\hline Stadtkreis & 0,142 & 0,111 & 0,000 \\
\hline Metropole & 0,217 & 0,279 & 0,000 \\
\hline Alter des Betriebs & 16,631 & 16,378 & 0,012 \\
\hline Schleswig-Holstein & 0,033 & 0,024 & 0,000 \\
\hline Hamburg & 0,011 & 0,021 & 0,000 \\
\hline Niedersachsen & 0,067 & 0,081 & 0,000 \\
\hline Bremen & 0,005 & 0,016 & 0,000 \\
\hline Nordrhein-Westfalen & 0,143 & 0,138 & 0,145 \\
\hline Hessen & 0,067 & 0,053 & 0,000 \\
\hline Rheinland-Pfalz & 0,034 & 0,024 & 0,000 \\
\hline Baden-Württemberg & 0,089 & 0,108 & 0,000 \\
\hline Bayern & 0,175 & 0,102 & 0,000 \\
\hline Saarland & 0,017 & 0,007 & 0,000 \\
\hline Berlin & 0,060 & 0,078 & 0,000 \\
\hline Brandenburg & 0,049 & 0,050 & 0,584 \\
\hline Mecklenburg-Vorpommern & 0,049 & 0,051 & 0,394 \\
\hline Sachsen & 0,094 & 0,114 & 0,000 \\
\hline
\end{tabular}


Tab. 5 (Fortsetzung)

\begin{tabular}{|c|c|c|c|}
\hline & Kontrollgruppe & Treatmentgruppe & $p$-Wert \\
\hline Sachsen-Anhalt & 0,052 & 0,062 & 0,000 \\
\hline Thüringen & 0,055 & 0,070 & 0,000 \\
\hline Arbeitslosenquote im Landkreis/Stadtkreis & 12,990 & 13,736 & 0,000 \\
\hline \multicolumn{4}{|l|}{ Beschäftigtenstruktur des Betriebs } \\
\hline Anteil Vollzeitkräfte & 0,801 & 0,817 & 0,000 \\
\hline Anteil geringfügig Beschäftigte & 0,076 & 0,054 & 0,000 \\
\hline Anteil sozialversicherungspflichtig beschäftigt & 0,924 & 0,946 & 0,000 \\
\hline Anteil Frauen & 0,156 & 0,117 & 0,000 \\
\hline Anteil kleine Teilzeit (<15 Stunden/Woche) & 0,068 & 0,048 & 0,000 \\
\hline Anteil große Teilzeit ( $\geq 15$ Stunden/Woche) & 0,022 & 0,015 & 0,000 \\
\hline Anteil ohne abgeschl. Berufsausbildung & 0,132 & 0,150 & 0,000 \\
\hline Anteil mit abgeschl. Berufsausbildung & 0,732 & 0,734 & 0,439 \\
\hline Anteil mit Hochschulabschluss & 0,028 & 0,007 & 0,000 \\
\hline Anteil Qualifizierung unbekannt & 0,107 & 0,110 & 0,276 \\
\hline Anteil Auszubildende & 0,102 & 0,113 & 0,000 \\
\hline Anteil Nicht-Facharbeiter & 0,092 & 0,107 & 0,000 \\
\hline Anteil Facharbeiter & 0,588 & 0,671 & 0,000 \\
\hline Anteil Angestellte & 0,186 & 0,078 & 0,000 \\
\hline Anteil Meister und Poliere & 0,029 & 0,029 & 0,815 \\
\hline Anteil einfache manuelle Berufe & 0,081 & 0,027 & 0,000 \\
\hline Anteil einfache Dienstleistungsberufe & 0,034 & 0,018 & 0,000 \\
\hline Anteil Haupterwerbskonzept & 0,987 & 0,991 & 0,000 \\
\hline Anteil 15-19 Jahre & 0,071 & 0,075 & 0,000 \\
\hline Anteil Beschäftigte 20-24 Jahre & 0,109 & 0,122 & 0,000 \\
\hline Anteil Beschäftigte 25-29 Jahre & 0,097 & 0,104 & 0,000 \\
\hline Anteil Beschäftigte 30-34 Jahre & 0,128 & 0,123 & 0,000 \\
\hline Anteil Beschäftigte 35 bis 39 Jahre & 0,158 & 0,151 & 0,000 \\
\hline Anteil Beschäftigte 40 bis 44 Jahre & 0,145 & 0,141 & 0,000 \\
\hline Anteil Beschäftigte 45 bis 49 Jahre & 0,107 & 0,098 & 0,000 \\
\hline Anteil Beschäftigte 50 bis 54 Jahre & 0,086 & 0,090 & 0,000 \\
\hline Anteil Beschäftigte 55 bis 59 Jahre & 0,054 & 0,056 & 0,000 \\
\hline Anteil Beschäftigte 60 bis 64 Jahre & 0,032 & 0,028 & 0,000 \\
\hline Anteil Beschäftigte über 65 Jahre & 0,015 & 0,011 & 0,000 \\
\hline \multicolumn{4}{|l|}{ Betriebsgröße } \\
\hline 1-5 Beschäftigte & 0,074 & 0,075 & 0,788 \\
\hline 6-10 Beschäftigte & 0,134 & 0,132 & 0,672 \\
\hline 11-20 Beschäftigte & 0,200 & 0,246 & 0,000 \\
\hline 21-100 Beschäftigte & 0,377 & 0,449 & 0,000 \\
\hline Über 100 Beschäftigte & 0,215 & 0,097 & 0,000 \\
\hline
\end{tabular}

Anmerkungen: Dargestellt sind die Mittelwerte der jeweiligen Variablen in der Treatmentgruppe und der Kontrollgruppe. Der $p$-Wert gibt das Signifikanzniveau eines Tests der Nullhypothese auf Gleichheit des Mittelwerts in den beiden Gruppen wieder. Quelle: IEB, BHP, eigene Berechnungen 


\section{Literatur}

Boockmann, B., Krumm, R., Rattenhuber, P., Neumann, M.: Turning the switch: an evaluation of the minimum wage in the German electrical trade using natural experiments. Mimeo, Institut für Angewandte Wirtschaftsforschung, Tübingen (2012)

Flinn, C.J.: The Minimum Wage and Labor Market Outcomes. MIT Press, Cambridge (2010)

Fitzenberger, B., Osikominu, A., Völter, R.: Imputation rules to improve the education variable in the IAB employment subsample. FDZ Methodenreport, 03/2005, Nürnberg (2005)

Hethey-Maier, T., Seth, S.: Das Betriebs-Historik-Panel (BHP) 19752008. FDZ Datenreport 4/2010, Nürnberg (2010)

IAB [Institut für Arbeitsmarkt- und Berufsforschung], RWI [Rheinisch-Westfälisches Institut für Wirtschaftsforschung], ISG [Institut für Sozialforschung und Gesellschaftspolitik]: Evaluation bestehender gesetzlicher Mindestlohnregelungen, Branche: Bauhauptgewerbe. Endbericht an das Ministerium für Arbeit und Soziales (2011)

IAW [Institut für Angewandte Wirtschaftsforschung]: Evaluation bestehender gesetzlicher Mindestlohnregelungen, Branche: Malerund Lackiererhandwerk. Endbericht an das Ministerium für Arbeit und Soziales, Tübingen (2011a)

IAW [Institut für Angewandte Wirtschaftsforschung]: Evaluation bestehender gesetzlicher Mindestlohnregelungen, Branche: Elektrohandwerk. Endbericht an das Ministerium für Arbeit und Soziales, Tübingen (2011b)

König, M., Möller, J.: Mindestlohneffekte des Entsendegesetzes? Eine Mikrodatenanalyse für die Deutsche Bauwirtschaft. Z. Arb.markt Forsch. 41(2/3), 327-346 (2008)

Lechner, M.: The estimation of causal effects by difference-indifference methods. University of St. Gallen Department of Economics discussion paper No. 2010-28 (2011)

Manning, A.: Monopsony in Motion: Imperfect Competition in Labor Markets. Princeton University Press, Princeton (2003)

Müller, K.-U.: Employment effects of a sectoral minimum wage in Germany. DIW discussion paper 1061, Berlin (2010)
Oberschachtsiek, D., Scioch, P., Seysen, C., Heining, J.: Stichprobe der Integrierten Erwerbsbiografien IEBS - Handbuch für die IEBS in der Fassung 2008. FDZ Datenreport, 03/2009, Nürnberg (2009)

Puhani, P.A.: The treatment effect, the cross difference, and the interaction term in nonlinear "difference-in-differences". Models, IZA discussion paper 3478, Bonn (2008)

Rattenhuber, P.: Building the minimum wage in Germany. Germany's first sectoral minimum wage and its impact on wages in the construction industry. DIW discussion paper 1111, Berlin (2011)

Bernhard Boockmann is managing director of the Institute for Applied Economic Research (IAW) and adjunct professor at the University of Tubingen. He studied economics and philosophy at the universities of Heidelberg and Mannheim and economics at University College London. From 1998 to 2007 he was a researcher at the Centre for European Economic Research (ZEW), Mannheim. He is a member of the standing committee on sociai policy of the German Economic Association and research fellow of the IZA network. His research centres on issues such as evaluation of labour market policies, the analysis of individual work histories, labour market regulations and wage determination.

Michael Neumann worked as a research associate at the Institute for Applied Economic Research (IAW) in Tubingen before he joined the DIW Graduate Center in 2011. He holds a degree in Economics from the University of Tubingen and spent an academic year at the University of Uppsala, Sweden. During his studies, Michael focused on quantitative methods of economic science and interned at the Institute for the Study of Labor (IZA) in Bonn and the Federal Ministry of Labor and Social Affairs.

Pia Rattenhuber holds a degree in International Economics from the University of Tubingen, a Masters in Economics from Universitat Pompeu Fabra, Spain, and a Ph.D. in Economics from Free University of Berlin. She has worked as a research associate at DIW Berlin and IAW Tubingen. Her research centers on the effects of minimum wages and tax policies on labor market outcomes. 